\title{
ON THE MODULE STRUCTURE OF THE CENTER OF HYPERELLIPTIC KRICHEVER-NOVIKOV ALGEBRAS II
}

\author{
BEN COX, XIANGQIAN GUO, MEE SEONG IM, AND KAIMING ZHAO
}

\begin{abstract}
Let $R:=R_{2}(p)=\mathbb{C}\left[t^{ \pm 1}, u: u^{2}=t\left(t-\alpha_{1}\right) \cdots\left(t-\alpha_{2 n}\right)\right]$ be the coordinate ring of a nonsingular hyperelliptic curve and let $\mathfrak{g} \otimes R$ be the corresponding current Lie algebra. Here $\mathfrak{g}$ is a finite dimensional simple Lie algebra defined over $\mathbb{C}$ and

$$
p(t)=t\left(t-\alpha_{1}\right) \cdots\left(t-\alpha_{2 n}\right)=\sum_{k=1}^{2 n+1} a_{k} t^{k} .
$$

In earlier work, Cox and Im gave a generator and relations description of the universal central extension of $\mathfrak{g} \otimes R$ in terms of certain families of polynomials $P_{k, i}$ and $Q_{k, i}$ and they described how the center $\Omega_{R} / d R$ of this universal central extension decomposes into a direct sum of irreducible representations when the automorphism group was the cyclic group $C_{2 k}$ or the dihedral group $D_{2 k}$. We give examples of $2 n$-tuples $\left(\alpha_{1}, \ldots, \alpha_{2 n}\right)$, which are the automorphism groups $\mathbb{G}_{n}=\operatorname{Dic}_{n}, \mathbb{U}_{n} \cong D_{n}(n$ odd $)$, or $\mathbb{U}_{n}(n$ even $)$ of the hyperelliptic curves
\end{abstract}

$$
S=\mathbb{C}\left[t, u: u^{2}=t\left(t-\alpha_{1}\right) \cdots\left(t-\alpha_{2 n}\right)\right]
$$

given in CGLZ17. In the work below, we describe this decomposition when the automorphism group is $\mathbb{U}_{n}=D_{n}$, where $n$ is odd.

\section{INTRODUCTION}

In CGLZ14 amongst other results, the automorphism group of derivation ring $\operatorname{Der}(R)$ for $R=\mathbb{C}\left[t,\left(t-a_{1}\right)^{-1}, \ldots,\left(t-a_{n}\right)^{-1}\right]$ was described and interestingly enough the five Kleinian groups $\mathbb{Z}_{n}, D_{n}, A_{4}, S_{4}$ and $A_{5}$ appear as automorphism groups of $R$ for particular choices of $a_{1}, \ldots, a_{n}$. These five groups also naturally appear in the McKay correspondence, tying together the representation theory of finite subgroups $G$ of $S L_{n}(\mathbb{C})$ to the resolution of singularities of quotient orbifolds $\mathbb{C}^{n} / G$. In this same paper, CGLZ14, the authors also described the universal central extension of this derivation KricheverNovikov algebra. One can in a straightforward manner show that the automorphism group acts on the center $\Omega_{R} / d R$ and one can naturally ask how the center decomposes into a direct sum of irreducible representations. This is exactly what described in [Cox16a].

In previous work (see [CI18) of the first and third authors we described how the center $\Omega_{R} / d R$ of the universal central extension of the hyperelliptic Lie algebra $\mathfrak{g} \otimes R$ with $R=\mathbb{C}\left[t, t^{-1}, u: u^{2}=p(t)\right]$ decomposes as a direct sum of irreducible modules for the corresponding automorphism groups $C_{n}$ and $D_{n}$, ( $n$ even). Here $p(t)=t(t-$ $\left.\alpha_{1}\right) \cdots\left(t-\alpha_{2 n}\right)=\sum_{i=1}^{2 n+1} a_{i} t^{i}$, with $\alpha_{i}$ pairwise distinct complex numbers. From the work of Grothendieck on the relationship between a Riemann surface and its de Rham 
cohomology groups one has

$$
\Omega_{R} / d R=\mathbb{C} \omega_{0} \oplus \ldots \oplus \mathbb{C} \omega_{2 n}
$$

where $\omega_{0}=\overline{t^{-1} d t}$ and $\omega_{i}=\overline{t^{-j} u d t}$ for $i=1, \ldots, 2 n$.

In CI18] we described how $\Omega_{R} / d R$ decomposes as a sum of irreducible representations for the groups $C_{2 k}$ and $D_{2 k}$ when $k \mid n$.

Using this theorem one can give a description of the bracket of two basis elements in the universal central extension of $\mathfrak{g} \otimes R$ in terms of polynomials $P_{k, i}$ and $Q_{k, i}$ defined recursively

$$
(2 k+r+3) P_{k, i}=-\sum_{j=1}^{r}(3 j+2 k-2 r) a_{j} P_{k-r+j-1, i} \quad \text { for all } k \geq 0
$$

with the initial conditions $P_{l, i}=\delta_{l, i},-r \leq i, l \leq-1$ and

$$
(2 m-3) a_{1} Q_{m, i}=\sum_{j=2}^{r+1}(3 j-2 m) a_{j} Q_{m-j+1, i} \quad \text { for all } m>0
$$

with the initial conditions $Q_{l, i}=\delta_{l,-i}$ for $1 \leq l \leq r$ and $-r \leq i \leq-1$. In this paper $\mathfrak{g}$ is assumed throughout to be a finite dimensional simple Lie algebra defined over the complex numbers. The generating series for these polynomials can be written in terms of hyperelliptic integrals using Bell polynomials and Faá de Bruno's formula (see Arb00, [Bel28], [FdB55] and [FdB57]). One can compare this result to that given in [Cox16b] and also in CZ17.

We also describe in this paper (see Theorem 4.3) how Kähler differentials modulo exact forms $\Omega_{R} / d R$ decompose under the action of the automorphism group of the coordinate $\operatorname{ring} R:=R_{2}(p)=\mathbb{C}\left[t^{ \pm 1}, u: u^{2}=p(t)\right]$, where $p(t)=t\left(t-\alpha_{1}\right) \cdots\left(t-\alpha_{2 n}\right)=\sum_{i=1}^{2 n+1} a_{i} t^{i}$, with the $\alpha_{i}$ being pairwise distinct roots. We recall Theorem 2.2 , where we describe the automorphism group of the universal central extension of the hyperelliptic Lie algebra as a $\mathbb{Z}_{2}$-graded Lie algebra. This corrects a small error in our previous work [CGLZ17].

The possible automorphism groups for the hyperelliptic curve

$$
R=\mathbb{C}\left[t^{ \pm 1}, u: u^{2}=t\left(t-\alpha_{1}\right) \cdots\left(t-\alpha_{2 n}\right)\right]
$$

are the groups $C_{2 k}=\mathbb{Z}_{2 k}$ or one of the groups

$$
\begin{aligned}
D_{2 k} & :=\left\langle x, y \mid x^{2 k}=1, y^{2}=1, x y x=y\right\rangle, \\
D_{i c_{k}} & :=\left\langle x, y \mid x^{2 k}=1, y^{2}=x^{k}, x y x=y\right\rangle, \\
\mathbb{U}_{k} & :=\left\langle x, y \mid x^{2 k}=1, y^{2}=1, x y x=y x^{k}\right\rangle .
\end{aligned}
$$

See Theorem 2.2 below, CGLZ17, Corollary 15], BGG93] and Sha03 for more detail.

The polynomials $P_{k, i}$ and $Q_{l, j}$, which are defined in Proposition 3.3 in [CI18], give us a description of how the center decomposes under the group of automorphisms of $R$. The automorphism group of $R$ has a natural action on $\Omega_{R} / d R$ and thus it is natural 
to wonder how this representation decomposes into a direct sum of irreducible representations. When the automorphism group is $C_{2 k}$, we can rewrite (2) as a direct sum of 1-dimensional irreducible $C_{2 k}$-representations. More precisely the center decomposes as:

$$
\Omega_{R} / d R \cong U_{0} \oplus \ldots \oplus U_{k-1}
$$

where $U_{r}=\bigoplus_{i \equiv r \bmod k, 1 \leq i \leq 2 n} \mathbb{C} \omega_{i}$ for $r=1, \ldots, k-1$, a sum of one-dimensional irreducible representation of $C_{2 k}$. The $U_{r}$ are irreducible representations with character $\chi_{r}(s)=$ $\exp (2 \pi \imath r s / 2 k)$ each occurring with multiplicity $l$ and

$$
U_{0}=\mathbb{C} \omega_{0} \oplus \bigoplus_{i=1}^{l} \mathbb{C} \omega_{k i}
$$

If the automorphism group is $D_{2 k}$, with $c^{2 n}=a_{1}, k \mid 2 n$, and $k$ is even, the center decomposes under the action of $D_{2 k}$ as

$$
\Omega_{R} / d R \cong \mathbb{C} \omega_{0} \oplus \bigoplus_{i=3}^{4} U_{i}^{\frac{\left(1-(-1)^{k}\right) n}{2 k}} \oplus \bigoplus_{h=1}^{k-1} V_{h}^{\oplus \frac{\left(1-(-1)^{h}\right) n}{k}}
$$

where $U_{i}, i=1,2,3,4$, are the irreducible one dimensional representations for $D_{2 k}$ with character $\rho_{i}$ and $V_{h}$ are the irreducible 2-dimensional representations for $D_{2 k}$ with character $\chi_{h}, 1 \leq h \leq k-1$. Here $\mathbb{C} \omega_{0}$ and $U_{1}$ are the trivial representation.

On the other hand if $k$ is odd, then the center decomposes as

$$
\Omega_{R} / d R \cong \mathbb{C} \omega_{0} \oplus \bigoplus_{i=3}^{4} U_{i}^{\oplus \Upsilon_{i}\left(\epsilon_{i}, \nu_{i}\right)} \oplus \bigoplus_{h=1}^{k-1} V_{h}^{\oplus \frac{\left(1-(-1)^{h}\right) n}{k}}
$$

where

$$
\Upsilon_{i}\left(\epsilon_{i}, \nu_{i}\right)=\frac{\left(1-(-1)^{k}\right) n}{2 k}\left(\delta_{i, 3}+\delta_{i, 4}\right)+(-1)^{i} \frac{1-(-1)^{n}}{4}+\frac{1}{2}(-1)^{i} \sum_{i=n+3}^{2 n} c^{n+3-2 i} P_{i-n-3,-i}
$$

This is all derived in [CI18, Theorem 7.2].

We now turn to the case when the automorphism group is $D_{n}$, where $n$ is odd. For $n$ odd, the center decomposes as

$$
\Omega_{R} / d R \cong \mathbb{C} \omega_{0} \oplus U_{1}^{\Xi_{1}} \oplus U_{2}^{\Xi_{2}} \oplus \bigoplus_{j=1}^{\frac{n-1}{2}} V_{j}
$$

where

$$
\Xi_{1}=\frac{1}{2}-\frac{1}{2} \sum_{i=n+3}^{2 n} c^{\frac{n+3-2 i}{2}} P_{i-n-3,-i} \quad \text { and } \quad \Xi_{2}=\frac{3}{2}+\frac{1}{2} \sum_{i=n+3}^{2 n} c^{\frac{n+3-2 i}{2}} P_{i-n-3,-i}
$$

$\mathbb{C} \omega_{0}$ is a 1-dimensional irreducible representation, $U_{i}$ are pairwise distinct 1-dimensional irreducible representations, and $V_{j}$ are pairwise distinct 2-dimensional irreducible representations (see Theorem 4.3 below). To prove this result below, we use the representation 
theory techniques of Frobenius and Schur as explained by Serre in [Ser77] and by Fulton and Harris in [FH91] to prove our results.

Since Dic $_{k}$ for $k$ gives the same character table as that for $D_{2 k}$ in our paper the decomposition theorem should nearly look the same with the exception of that the irreducible representations that appear are different and are for different groups. In other words, the multiplicities of the irreducibles are the same but the representations are different. We will explore this in our future work.

\section{Automorphism group For $R=\mathbb{C}\left[t^{ \pm 1}, u: u^{2}=p(t)=t\left(t-\alpha_{1}\right) \cdots\left(t-\alpha_{2 n}\right)\right]$.}

In this section, we restrict to the case of $r=2 n$ which allows us to use the results in [CGLZ17], [BGG93] and [Sha03] on automorphism groups of such algebras.

2.1. Automorphisms of the algebra $R_{2}(p)$. Let $S_{2 n}$ be the symmetry group on the finite set $\{1,2, \ldots, 2 n\}$.

First we recall some background material.

Theorem 2.1 ([BGG93] and [Sha03]). The automorphism group of a hyperelliptic curve $A=\mathbb{C}\left[X, Y \mid Y^{2}=P(X)\right]$ is isomorphic to one of the following groups:

$$
D_{n}, \quad \mathbb{Z}_{n}, \quad \mathbb{V}_{n}, \quad \mathbb{H}_{n}, \quad \mathbb{G}_{n}, \quad \mathbb{U}_{n}, \quad G L_{2}(3), \quad W_{2}, \quad W_{3}
$$

where

$$
\begin{aligned}
\mathbb{V}_{n} & :=\left\langle x, y \mid x^{4}, y^{n},(x y)^{2},\left(x^{-1} y\right)^{2}\right\rangle, \\
\mathbb{H}_{n} & :=\left\langle x, y \mid x^{4}, y^{2} x^{2},(x y)^{n}\right\rangle, \\
\mathbb{G}_{n} & :=\left\langle x, y \mid x^{2} y^{n}, y^{2 n}, x^{-1} y x y\right\rangle, \\
\mathbb{U}_{n} & :=\left\langle x, y \mid x^{2}, y^{2 n}, x y x y^{n+1}\right\rangle, \\
W_{2} & :=\left\langle x, y \mid x^{4}, y^{3}, y x^{2} y^{-1} x^{2},(x y)^{4}\right\rangle, \\
W_{3} & :=\left\langle x, y \mid x^{4}, y^{3}, x^{2}(x y)^{4},(x y)^{8}\right\rangle .
\end{aligned}
$$

In [Sha03] a description of the reduced automorphism group is described for a given polynomial $P(X)$. In our paper we do not work with the reduced automorphism group and our coordinate ring is the localization $\mathbb{C}\left[t, t^{-1}, u: u^{2}=p(t)=t\left(t-\alpha_{1}\right) \cdots\left(t-\alpha_{2 n}\right)\right]$ of $A$.

For convenience, we need the following alternative description of the group $\mathbb{U}_{n}$ :

$$
\mathbb{U}_{n}=\left\langle x_{1}, y_{1}: y_{1}^{2 n}=1, x_{1}^{2}=y_{1}^{n}, y_{1} x_{1} y_{1}=x_{1}^{-1}\right\rangle \text {. }
$$

Indeed, setting $y=y_{1}$ and $x=x_{1} y_{1}$, we can get $y^{2 n}=1, x^{2}=x_{1} y_{1} x_{1} y_{1}=1$ and $x y x y^{n+1}=x_{1} y_{1}^{2} x_{1} y_{1}^{n+2}=y_{1}^{-1}\left(y_{1} x_{1} y_{1}\right)^{2} y_{1}^{n+1}=y_{1}^{-1} x_{1}^{-2} y_{1}^{n+1}=1$, which is just the generating relations of $\mathbb{U}_{n}$ in Theorem [2.1. On the other hand, if $x, y$ are the generators of $\mathbb{U}_{n}$ as described in Theorem 2.1, we set $y_{1}=y$ and $x_{1}=x y$, which gives $y_{1}^{2 n}=1$, $x_{1}^{2}=x y x y=y^{n}=y_{1}^{n}$ and $y_{1} x_{1} y_{1}=y x y y=x y^{n+1}=x_{1} y_{1}^{n}$, the generating relations in (8) . So the group $\mathbb{U}_{n}$ defined by (8) coincides with the group $\mathbb{U}_{n}$ in Theorem 2.1 .

The result below describes the automorphisms of the algebra of the superelliptic curve $u^{2}=p(t)$, and corrects some errors that occur in [CGLZ17], Corollary 15. 
Theorem 2.2 (Corollary 15, CGLZ17]). Let $p(t)=t\left(t-\alpha_{1}\right) \cdots\left(t-\alpha_{2 n}\right)$, where $\alpha_{i}$ are pairwise distinct nonzero roots. The only possible automorphisms $\phi \in \operatorname{Aut}\left(R_{2}(p)\right)$ of the algebra $R_{2}(p)$ are of the following types:

(1) There exist some $4 n$-th root of unity $\xi$ and $\gamma \in S_{2 n}$ with $\alpha_{\gamma(i)}=\xi^{2} \alpha_{i}$ such that

$$
\phi(t)=\xi^{2} t, \quad \phi(u)=\xi u .
$$

We denote this $\phi$ by $\phi_{\xi}$ which satisfies $\left(\phi_{\xi}\right)^{4 n}=i d$. Note that $\gamma$ is uniquely determined by $\xi^{2}$.

(2) There exists $c \in \mathbb{C}$ and $\gamma \in S_{2 n}$ with $\alpha_{i} \alpha_{\gamma(i)}=c^{2}$ such that

$$
\phi(t)=c^{2} t^{-1}, \phi(u)=\epsilon c^{n+1} t^{-n-1} u,
$$

where $\epsilon= \pm 1$ if $\prod_{i=1}^{2 n} \alpha_{i}=c^{2 n}$ and $\epsilon= \pm \imath$ if $\prod_{i=1}^{2 n} \alpha_{i}=-c^{2 n}$. We denote these $\phi$ by $\psi_{c}^{ \pm}$respectively which satisfy $\left(\psi_{c}^{ \pm}\right)^{2}=$ id if $\epsilon= \pm 1 ;\left(\psi_{c}^{ \pm}\right)^{2}=\phi_{-1}$ if $\epsilon= \pm \imath$; $\psi_{c}^{-}=\psi_{-c}^{+}$if $n$ is even; and $\psi_{-c}^{ \pm}=\psi_{c}^{ \pm}$if $n$ is odd. Note that $\gamma$ is uniquely determined by $c^{2}$.

For convenience we denote $\psi_{c}=\psi_{c}^{+}$in Theorem $2.2(2)$, and let $\operatorname{Aut}_{1}\left(R_{2}(p)\right)$ be the set of all automorphisms coming from $2.2(1)$, which is a subgroup of $\operatorname{Aut}\left(R_{2}(p)\right)$.

Corollary 2.3 ([CGLZ17], Corollary 16). Let $p(t)=t\left(t-\alpha_{1}\right) \cdots\left(t-\alpha_{2 n}\right)$, with pairwise distinct roots.

(1) There exists some $k \in \mathbb{N}$ with $k \mid 2 n$ such that $\operatorname{Aut}_{1}\left(R_{2}(p)\right)$ is generated by an automorphism $\phi_{\xi}$ of order $2 k$, where $\xi$ is any primitive root of unity of order $2 k$.

(2) If $\psi_{c}$ does not exist in $\operatorname{Aut}\left(R_{2}(p)\right)$ for any nonzero complex number $c$, then

$$
\operatorname{Aut}\left(R_{2}(p)\right)=\operatorname{Aut}_{1}\left(R_{2}(p)\right)=\left\langle\phi_{\xi}\right\rangle \simeq \mathbb{Z}_{2 k},
$$

where $k$ and $\xi$ are as in (1).

(3) If $\psi_{c}$ exists in $\operatorname{Aut}\left(R_{2}(p)\right)$ for some complex number $c$ with $c^{4 n}=\prod_{i=1}^{2 n} \alpha_{i}$, then

$$
\operatorname{Aut}\left(R_{2}(p)\right)=\left\{\phi_{\xi}^{i}, \psi_{c} \phi_{\xi}^{i}: i=0,1, \ldots, 2 k-1\right\}
$$

where $\phi_{\xi} \in \operatorname{Aut}_{1}\left(R_{2}(p)\right)$ is an automorphism of order $2 k$ as in (11). In particular, we have $\left|\operatorname{Aut}\left(R_{2}(p)\right)\right|=4 k$. Moreover we have:

$$
\operatorname{Aut}\left(R_{2}(p)\right)= \begin{cases}D_{k} & \text { if } 2 n / k \text { is even and } \prod_{i=1}^{2 n} \alpha_{i}=c^{2 n}, \\ \mathbb{G}_{k} & \text { if } 2 n / k \text { is even and } \prod_{i=1}^{2 n} \alpha_{i}=-c^{2 n}, \\ \mathbb{U}_{k} \text { with } k \text { even } & \text { if } 2 n / k \text { is odd. }\end{cases}
$$

Proof. For any $\phi_{\xi}, \phi_{\xi^{\prime}} \in \operatorname{Aut}\left(R_{2}(p)\right), \xi, \xi^{\prime} \in \mathbb{C}$, we have $\phi_{\xi} \phi_{\xi^{\prime}}=\phi_{\xi \xi^{\prime}}$ and hence $\operatorname{Aut}_{1}\left(R_{2}(p)\right)$ is a finite abelian group. Let $\phi_{\xi}$ be an automorphism of the greatest order $2 k$ with $k \mid 2 n$. Then any other automorphism $\phi_{\xi^{\prime}}$ must have order $k^{\prime}$ with $k^{\prime} \mid k$. Hence $\xi^{\prime}=\xi^{r}$ for some $r \in \mathbb{N}$ and $\phi_{\xi^{\prime}}=\phi_{\xi}^{r}$. Now $\operatorname{Aut}_{1}\left(R_{2}(p)\right)$ is generated by $\phi_{\xi}$. Hence (1) and (2) follow.

To prove (3), we first note that $\psi_{c} \psi_{c^{\prime}} \in \operatorname{Aut}_{1}\left(R_{2}(p)\right)$ for any $\psi_{c}, \psi_{c^{\prime}} \in \operatorname{Aut}\left(R_{2}(p)\right)$, where $c, c^{\prime} \in \mathbb{C}$. Let $\xi, \phi_{\xi}$ and $k$ be as in (10), then $\xi$ is a primitive root of unity of order $2 k$ and hence $\xi^{k}=-1$. Set $l=2 n / k$. Take any $\psi_{c} \in \operatorname{Aut}\left(R_{2}(p)\right)$. Now we can deduce (11) easily. Noticing that elements listed in (11) are pairwise distinct, we see $\left|\operatorname{Aut}\left(R_{2}(p)\right)\right|=4 k$. 
With straightforward computations we deduce that

$$
\left(\phi_{\xi} \psi_{c} \phi_{\xi}\right)(t)=\psi_{c}(t) \text { and }\left(\phi_{\xi} \psi_{c} \phi_{\xi}\right)(u)=\xi^{-2 n} \psi_{c}(u)=(-1)^{l} \psi_{c}(u)
$$

In case $l$ is even, we have $\phi_{\xi} \psi_{c} \phi_{\xi}=\psi_{c}$. If $\prod_{i=1}^{2 n} \alpha_{i}=c^{2 n}$, then $\psi_{c}^{2}=\mathrm{id}$ and hence we have an epimorphism from $D_{2 k}$ to $\operatorname{Aut}\left(R_{2}(p)\right)$. Since $\left|D_{2 k}\right|=4 k$, we have $\operatorname{Aut}\left(R_{2}(p)\right) \cong D_{2 k}$. Similarly, if $\prod_{i=1}^{2 n} \alpha_{i}=-c^{2 n}$, then $\psi_{c}^{2}=\phi_{-1}=\phi_{\xi}^{k}$ and we have an epimorphism from $\mathbb{G}_{k}$ to $\operatorname{Aut}\left(R_{2}(p)\right)$. Moreover, from the fact $\left|\mathbb{G}_{k}\right| \leq 4 k$ we obtain that $\operatorname{Aut}\left(R_{2}(p)\right) \cong \mathbb{G}_{k}=\operatorname{Dic}_{k}$.

In case $l$ is odd, we have $\phi_{\xi} \psi_{c} \phi_{\xi}=\psi_{c} \phi_{-1}=\psi_{c} \phi_{\xi}^{k}$. If $\prod_{i=1}^{2 n} \alpha_{i}=c^{2 n}$, then $\psi_{c}^{2}=\mathrm{id}$ and $\psi_{c}, \phi_{\xi}$ satisfy the generating relations for $\mathbb{U}_{k}$ in Theorem 2.1; if $\prod_{i=1}^{2 n} \alpha_{i}=-c^{2 n}$, then $\psi_{c}^{2}=\phi_{-1}=\phi_{\xi}^{k}$ and $\psi_{c}, \phi_{\xi}$ satisfy the generating relations for $\mathbb{U}_{k}$ in (8) . We get an epimorphism from $\mathbb{U}_{k}$ ( $k$ even) to $\operatorname{Aut}\left(R_{2}(p)\right)$, which is an isomorphism by a similar argument as in the previous paragraph.

In the next example we will realize the groups $\operatorname{Dic}_{n}=\mathbb{G}_{n}$ and $\mathbb{U}_{n}$.

Example 2.4. Let $p(t)=t \prod_{i=1}^{l} \prod_{j=1}^{k}\left(t-c_{i} \xi^{2 j}\right)$ with pairwise distinct roots, where $\xi$ is primitive root of unity of order $2 k$ and $l=2 n / k$. Set $\alpha_{(i-1) k+j}=c_{i} \xi^{2 j}$. We have $\phi_{\xi} \in \operatorname{Aut}_{1}\left(R_{2}(p)\right)$ which has order $2 k$.

(1) Suppose that $l=3$ and $\left|c_{1}\right|,\left|c_{2}\right|,\left|c_{3}\right|$ are pairwise distinct. We see that $\operatorname{Aut}_{1}\left(R_{2}(p)\right)$ $=\left\langle\phi_{\xi}\right\rangle \cong \mathbb{Z}_{2 k}$. But we can not find $c \in \mathbb{C}$ and $\gamma \in S_{2 n}$ such that $\alpha_{i} \alpha_{\gamma(i)}=c^{2}$ (i.e., $|c|^{2}=\left|c_{i}\right| \cdot\left|c_{i^{\prime}}\right|$ for all $i=1,2,3$ where $i^{\prime} \neq i$ is determined by $i$ ). So there does not exist automorphism of $R_{2}(p)$ coming from (2) of Lemma 2.2. Hence $\operatorname{Aut}\left(R_{2}(p)\right)=\operatorname{Aut}_{1}\left(R_{2}(p)\right) \cong \mathbb{Z}_{2 k}$.

(2) Suppose that $l=2$ and $\left|c_{2}\right| \neq\left|c_{1}\right|$. Then $k=n$, $\xi^{n}=-1$ and $\prod_{i=1}^{2 n} \alpha_{i}=c_{1}^{n} c_{2}^{n}$. Take any $c \in \mathbb{C}$ such that $c^{2}=c_{1} c_{2}$, we have $\prod_{i=1}^{2 n} \alpha_{i}=c^{2 n}$ and

$$
c_{1} \xi^{j-i} c_{2} \xi^{j+i}=\left(\xi^{j} c\right)^{2}, \quad \prod_{i=1}^{2 n} \alpha_{i}=\left(\xi^{j} c\right)^{2 n} .
$$

We can define the automorphisms $\psi_{\xi^{j} c}^{ \pm}$for all $j=0,1, \ldots, 2 n-1$, that is,

$$
\psi_{\xi^{j} c}^{ \pm}: t \mapsto \xi^{2 j} c^{2} t^{-1}, \quad \phi(u)= \pm \xi^{j(n+1)} c^{n+1} t^{-n-1} u
$$

Denote $\psi_{c}=\psi_{c}^{+}$. We can check that $\psi_{\xi^{j} c}^{+}=\psi_{c} \phi_{\xi}^{j}$ if $j$ is even and $\psi_{\xi^{j} c}^{-}=\psi_{c} \phi_{\xi}^{j}$ if $j$ is odd. Moreover, $\psi_{\xi^{j} c}^{-}=\psi_{\xi^{n+j} c}^{+}$if $n$ is even and $\psi_{\xi^{j} c}^{ \pm}=\psi_{\xi^{n+j} c}^{ \pm}$if $n$ is odd. So these $\psi_{\xi^{j} c}^{ \pm}$give rise to exactly $2 n$ distinct automorphisms. By Corollary [2.3, we have

$$
D_{n} \cong \operatorname{Aut}\left(R_{2}(p)\right)=\left\{\phi_{\xi}^{j}, \psi_{c} \phi_{\xi}^{j} \mid j=0,1, \ldots, 2 n-1\right\} .
$$

This provides a realization of the group $D_{n}$.

(3) Suppose that $l=1, k=2 n$. Denote $c=\xi c_{1}$. Without loss of generality, we may assume $\xi^{n}=\imath$. Noticing $\xi^{2 n}=-1$, we have $\prod_{i=1}^{2 n} \alpha_{i}=c^{2 n}$ and

$$
\left(c \xi^{j+i}\right)\left(c \xi^{j-i}\right)=\left(\xi^{j} c\right)^{2}, \quad \prod_{i=1}^{2 n} \alpha_{i}= \begin{cases}\left(\xi^{j} c\right)^{2 n} & \text { if } j \text { is even }, \\ -\left(\xi^{j} c\right)^{2 n} & \text { if } j \text { is odd. }\end{cases}
$$


We can define $\psi_{\xi^{j} c}^{ \pm}$for $j=0,1, \ldots, 4 n-1$. More explicitly, we have

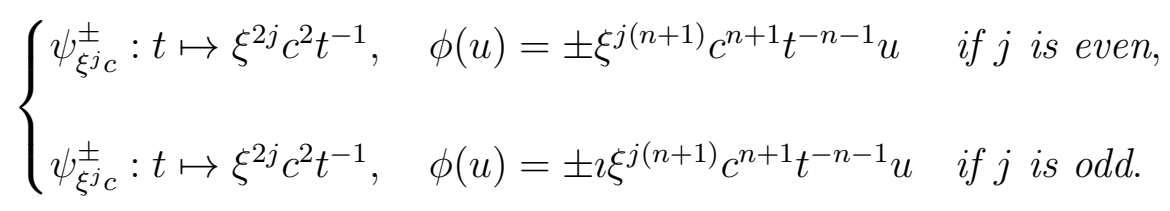

Denote $\psi_{c}=\psi_{c}^{+}$. We can check that $\psi_{\xi^{j} c}^{+}=\psi_{c} \phi_{\xi}^{j}$ if $j \equiv 0 \bmod 4$ or $j \equiv 3 \bmod 4$ and $\psi_{\xi^{j} c}^{-}=\psi_{c} \phi_{\xi}^{j}$ if $j \equiv 2 \bmod 4$ or $j \equiv 1 \bmod 4$. Moreover, $\psi_{\xi^{j} c}^{-}=\psi_{\xi^{2 n+j} c}^{+}$if $n$ is even and $\psi_{\xi^{j} c}^{ \pm}=\psi_{\xi^{2 n+j_{c}}}^{ \pm}$if $n$ is odd. So these $\psi_{\xi^{j} c}^{ \pm}$give rise to exactly $4 n$ distinct automorphisms. Using Corollary 2.3, we can write

$$
\mathbb{U}_{n} \cong \operatorname{Aut}\left(R_{2}(p)\right)=\left\{\phi_{\xi}^{j}, \psi_{c} \phi_{\xi}^{j}: j=0,1, \ldots, 4 n-1\right\} .
$$

This provides a realization of the group $\mathbb{U}_{n}$.

By the above example, we can deduce the following description of the groups Dic $_{n}=$ $\mathbb{G}_{n}, \mathbb{U}_{n}, \mathbb{V}_{2 n}$.

Corollary 2.5. Let $n$ be any positive integer. Then

(1) $\operatorname{Dic}_{n}=\mathbb{G}_{n}=\left\langle x, y: y^{2 n}=1, x^{2}=y^{n}, y x y=x\right\rangle=\left\{y^{i}, x y^{i}: i=0,1, \ldots, 2 n-1\right\}$,

(2) If $n$ is odd, then $\mathbb{U}_{n} \cong D_{n}$. If $n$ is even, then $\left|\mathbb{U}_{n}\right|=4 n$ and moreover

$$
\mathbb{U}_{n}=\left\langle x, y: y^{2 n}=1, x^{2}=1, y x y=x y^{n}\right\rangle=\left\{y^{i}, x y^{i}: i=0,1, \ldots, 2 n-1\right\} .
$$

Proof. Assertion (11) and the second statement of (2) follow directly from Corollary 2.3 and the above example. To prove the first statement of (2), let $x, y$ be the generators of $\mathbb{U}_{n}$ as described in (2). Then we have $y x=x y^{n-1}$, and hence $y^{n} x=x y^{n(n-1)}=x$ since $n$ is odd. We get $y^{n}=1$ and it follows that $\mathbb{U}_{n} \cong D_{n}$ if $n$ is odd.

We add to this another

Corollary 2.6 ([CI18], Corollary 6.4). Let $p(t)=t\left(t-\alpha_{1}\right) \cdots\left(t-\alpha_{2 n}\right)$, where $\alpha_{i}$ are distinct roots. Two possible types of automorphisms $\phi \in \operatorname{Aut}\left(R_{2}(p)\right)$ of the algebra $R_{2}(p)$ are the following:

(1) If $\alpha_{\gamma(i)}=\zeta \alpha_{i}$ for some $2 n$-th root of unity $\zeta$ and $\gamma \in S_{2 n}$, then

$$
\phi(t)=\zeta t, \quad \phi(u)= \pm \xi u
$$

where we can take $\xi=\zeta^{1 / 2}=\exp (2 \pi \imath / 2 k)$ with $\zeta$ having order $k$ and $k \mid 2 n$. It follows that $\phi$ has order $2 k$. In particular, after a change in indices

$$
\begin{aligned}
p(t) & =t\left(t-\alpha_{1}\right)\left(t-\zeta \alpha_{1}\right) \cdots\left(t-\zeta^{k-1} \alpha_{1}\right) \cdots\left(t-\alpha_{2 n / k}\right) \cdots\left(t-\zeta^{k-1} \alpha_{2 n / k}\right) \\
& =t\left(t^{k}-\alpha_{1}^{k}\right)\left(t^{k}-\alpha_{2}^{k}\right) \cdots\left(t^{k}-\alpha_{2 n / k}^{k}\right) \\
& =\sum_{q=0}^{\frac{2 n}{k}}(-1)^{q} e_{q}\left(\alpha_{1}^{k}, \ldots, \alpha_{2 n / k}^{k}\right) t^{2 n-q k+1},
\end{aligned}
$$


where $e_{q}\left(x_{1}, x_{2}, \ldots, x_{2 n / k}\right)$ is the elementary symmetric polynomial of degree $q$ in $x_{1}, \ldots, x_{2 n / k}$ :

$$
e_{q}\left(x_{1}, x_{2}, \ldots, x_{2 n / k}\right)=\sum_{1 \leq j_{1}<j_{2}<\ldots<j_{q} \leq 2 n / k} x_{j_{1}} x_{j_{2}} \cdots x_{j_{q}} .
$$

In this case, $\left\langle\phi_{\xi}^{+}\right\rangle \cong \mathbb{Z}_{2 k}$.

(2) If, in addition to the above, there exists $\beta \in S_{2 n}$ such that $\alpha_{i} \alpha_{\beta(i)}=c^{2}$ for all $i$, then $\phi_{\xi}^{ \pm}(t)=\zeta$ t and $\phi_{\xi}^{ \pm}(u)= \pm \xi u$, and $\psi(t)=c^{2} t^{-1}$ and

$$
\psi_{c}^{ \pm}(u)= \pm t^{-n-1} c^{n+1} u \quad \text { if } a_{1}=\prod_{i=1}^{2 n} \alpha_{i}=c^{2 n},
$$

or

$$
\psi_{c}^{ \pm}(u)= \pm t^{-n-1} \imath c^{n+1} u \quad \text { if } a_{1}=\prod_{i=1}^{2 n} \alpha_{i}=-c^{2 n} .
$$

In this case

$$
p(t)=\sum_{r=1}^{2 n+1} a_{r} t^{r}, \quad \text { where } \quad a_{k}= \pm c^{2 n-2 k+2} a_{2 n+2-k}
$$

for $k=1, \ldots, 2 n+1$. Here the \pm in (15) corresponds to the \pm in $a_{1}= \pm c^{2 n}$.

From [Skr88], we know that for any automorphism $\phi$ of the associative algebra $R_{2}(p)$, one obtains an automorphism $\tau$ of the Lie algebra $\mathcal{R}_{2}(p):=\operatorname{Der}\left(R_{2}(p)\right)$ through the equation

$$
\tau(f(t) \partial)=\phi(f)\left(\phi \circ \partial \circ \phi^{-1}\right) \quad \text { for all } f \in R_{2}(p) .
$$

In addition, any Lie algebra automorphism of $\mathcal{R}_{2}(p)$ can be obtained from (16). Denote by $\tau_{\zeta}^{ \pm}$and $\sigma_{c}^{ \pm}$the Lie algebra automorphisms corresponding to the associative algebra automorphisms $\phi_{\zeta}^{ \pm}$and $\psi_{c}^{ \pm}$in Theorem 2.2 (11) and (2) respectively (if they indeed exist). For convenience, denote $\tau_{\zeta}:=\tau_{\zeta}^{+}$and $\sigma_{c}:=\sigma_{c}^{+}$.

\section{Character Tables}

\subsection{Character Table and Irreducible Finite Dimensional Representations of} $\mathbb{U}_{n}$. Recall

$$
\mathbb{U}_{n}=\left\langle x, y \mid x^{2}, y^{2 n}, x y x y^{n+1}\right\rangle .
$$

Proposition 3.1.1. If $n$ is odd, then $\mathbb{U}_{n}=D_{n}$ is the dihedral group.

Suppose $n=2(2 s+1)$. The conjugacy classes of $\mathbb{U}_{n}$ are

$$
\begin{gathered}
\{1\}, \quad\left\{y^{2 s+1}\right\}, \quad\left\{y^{n}\right\}, \quad\left\{y^{6 s+3}\right\}, \\
\left\{y^{2 j}, y^{-2 j}\right\} \quad 1 \leq j<2 s+1=n / 2, \quad\left\{y^{2 j+1}, y^{n-2 j-1}\right\}, \quad\left\{y^{n+2 j+1}, y^{2 n-2 j-1}\right\} \quad 1 \leq 2 j+1<l=n / 2, \\
\left\{x, x y^{4}, \ldots, x y^{2 n-4}\right\}, \quad\left\{x y, x y^{5}, \ldots, x y^{2 n-3}\right\}, \quad\left\{x y^{2}, x y^{6}, \ldots, x y^{2 n-2}\right\} \\
\left\{x^{3}, x y^{7}, \ldots, x y^{2 n-1}\right\}
\end{gathered}
$$


for a total of $n+6$ conjugacy classes.

Suppose now $n=4 s$. The conjugacy classes of $\mathbb{U}_{n}$ are then

$$
\begin{gathered}
\{1\}, \quad\left\{y^{2 l}\right\}, \\
\left\{y^{2 j}, y^{-2 j}\right\} \quad \begin{array}{c}
1 \leq j<l, \quad\left\{y^{2 j+1}, y^{n-2 j-1}\right\}, \quad\left\{y^{n+2 j+1}, y^{2 n-2 j-1}\right\} \quad 1 \leq 2 j+1<l, \\
\left\{x, x y^{2}, \ldots, x y^{2 n-2}\right\}, \quad\left\{x y, x y^{3}, \ldots, x y^{2 n-1}\right\}
\end{array}
\end{gathered}
$$

for a total of $n+3$ conjugacy classes.

Proof. First we make the observation for $n$ odd. We have $y x=x y^{n-1}$ as $x^{2}=1$, $x y x y^{n+1}=1$ and $y^{2 n}=1$. Hence $y^{2 k+1} x=x y^{(2 k+1)(n-1)}=x y^{n-1-2 k}$. Thus if $n=2 l+1$ is odd we have $y^{n} x=y^{2 l+1} x=x y^{n-2 l-1}=x$. But then $y^{n}=1$. Thus our generators and relations would be

$$
x^{2}=1, \quad y^{n}=1, \quad x y x=y^{-1},
$$

and $\mathbb{U}_{n}=D_{n}$ is the dihedral group.

For the rest of the proof, we assume $n=2 l$ is even. We have $y^{k} x=x y^{k(n-1)}$ so

$$
x y^{2 j} x=y^{2 j(n-1)}=y^{-2 j}
$$

and

$$
x y^{2 j+1} x=y^{(2 j+1)(n-1)}=y^{n-2 j-1} .
$$

Thus we have two element conjugacy classes

$$
\left\{y^{2 j}, y^{2 n-2 j}\right\}, \quad\left\{y^{2 j+1}, y^{n-2 j-1}\right\}
$$

provided $y^{2 j} \neq y^{2 n-2 j}$ and $y^{2 j+1} \neq y^{n-2 j-1}$. For $1 \leq j<n / 2$ one has $y^{2 j} \neq y^{2 n-2 j}$ and we get all of the distinct such conjugacy classes if $1 \leq j<n / 2$.

We break this up into two different cases $n=2(2 s+1)$ and $n=4 s$, where $l=2 s+1$ or $l=2 s$, respectively.

Case when $n=2(2 s+1)$. For $y^{2 j+1}=y^{n-2 j-1}$, we have $4 j+2 \equiv n \bmod 2 n$ so that $2(2 j+1)=(2 k+1) n$ for some $k$. Hence $n$ must be even so $n=2 l$. Now $2 j+1=$ $(2 k+1) l$ and $l$ must be odd. Moreover $1 \leq 2 j+1=(2 k+1) l<2 n=4 l$. This implies $2 k+1 \in\{1,3\}$ or $2 j+1=l$ or $2 j+1=3 l$. If $n=2 l$, then for $2 j+1=l$ or $3 l$ we get $y^{n-2 j-1}=y^{2 l-l}=y^{l}=y^{2 j+1}$ and $y^{n-2 j-1}=y^{2 l-3 l}=y^{-l}=y^{3 l}=y^{2 j+1}$ in the later case as $y^{4 l}=y^{2 n}=1$.

Consider now the case $n=2 \mathrm{l}$ even with $l=2 s+1$ odd and $n>4$. We have the set of elements

$$
\left\{\underline{1}, y, \widehat{y^{2}}, \ldots, \frac{y^{2 j+1}}{y^{n+2 r+1}}, \ldots, \underline{y^{l}}, \ldots, \underline{y^{2 l-2 j-1}}, \ldots, \overline{y^{2 n-2 r-1}}, \ldots, \underline{y^{2 l}}, \ldots\right.
$$

that breaks up into conjugacy classes with one element in them (the underlined elements above):

$$
\{1\}, \quad\left\{y^{l}\right\}, \quad\left\{y^{2 l}=y^{n}\right\}, \quad\left\{y^{3 l}\right\},
$$


the conjugacy classes with even exponents $\left\{y^{2 j}, y^{2 n-2 j}\right\}$ with $1 \leq j<l=n / 2$ (the hatted elements above) and the other conjugacy classes with $y$ having an odd positive exponent are of the form

$$
\left\{y^{2 j+1}, y^{n-2 j-1}\right\} \quad \text { for } 1 \leq 2 j+1<l \text {, }
$$

and

$$
\left\{y^{n+2 j+1}, y^{2 n-2 j-1}\right\} \quad \text { for } 1 \leq 2 j+1<l
$$

(the boxed and over lined elements above). There are $l-1=(n / 2)-1$ of the latter two types of conjugacy classes.

Using the identities $x^{2}=y^{2 n}=1=x y x y^{n+1}$ we get $y x=x y^{n-1}$ and $y^{r} x=x y^{r(n-1)}$. Hence

$$
y^{r} x y^{k} y^{-r}=x y^{k+r(n-1)-l}=x y^{k+r(n-2)}
$$

and for $r=2 q+1$ we get

$$
y^{r} x y^{k} y^{-r}=x y^{k+(2 q+1)(n-2)}=x y^{k-4 q+n-2} .
$$

Consequently recalling $n=2(2 a+1)$ we get $y^{r} x y^{k} y^{-r}=x y^{k+4(a-q)}$. Moreover

$$
x\left(x y^{k}\right) x=y^{k} x=x y^{k(n-1)}=x y^{k(4 a+1)}=x y^{k+4 a k} .
$$

This implies that the set of elements

$$
\left\{x, x y, x y^{2}, \ldots, x y^{2 n-1}\right\}
$$

breaks up into four distinct conjugacy classes

$$
\left\{x, x y^{4}, \ldots x y^{2 n-4}\right\}, \quad\left\{x y, x y^{5}, \ldots x y^{2 n-3}\right\}, \quad\left\{x y^{2}, x y^{6}, \ldots x y^{2 n-2}\right\},
$$

and

$$
\left\{x^{3}, x y^{7}, \ldots x y^{2 n-1}\right\}
$$

Thus in the case of $n=2(2 l+1)$ there are exactly $n+6$ conjugacy classes.

Case when $n=4 s$. Now consider the case of $l=2 s$ so $n=4 s$. We can divide

$$
\left\{\underline{1}, y, \widehat{y^{2}}, \ldots, y^{2 j+1}, \ldots, y^{2 l-2 j-1}, \ldots, \underline{y^{2 l}}, \ldots \overline{y^{n+2 r+1}}, \ldots, \overline{y^{2 n-2 r-1}}, \ldots, \widehat{y^{2 n-2}}, y^{2 n-1}\right\}
$$

into conjugacy classes with one element in them (the underlined elements above):

$$
\{1\}, \quad\left\{y^{2 l}=y^{n}\right\}
$$

the conjugacy classes with even exponents $\left\{y^{2 j}, y^{2 n-2 j}\right\}$ with $1 \leq j<l=n / 2$ (the hatted elements above) and the other conjugacy classes with $y$ having an odd positive exponent are of the form

$$
\left\{y^{2 j+1}, y^{n-2 j-1}\right\} \quad \text { for } 1 \leq 2 j+1<l
$$

and

$$
\left\{y^{n+2 j+1}, y^{2 n-2 j-1}\right\} \quad \text { for } 1 \leq 2 j+1<l
$$

(the boxed and over lined elements above). There are $2(l / 2)=n / 2$ of the latter two types of conjugacy classes.

We lastly want to break

$$
\left\{x, x y, x y^{2}, \ldots, x y^{2 n-1}\right\}
$$


into distinct conjugacy classes. For this we observe

$$
y^{r} x y^{k} y^{-r}=x y^{k+r(n-2)}=x y^{k+4 r s-2 r}
$$

and

$$
x\left(x y^{k}\right) x=y^{k} x=x y^{k(n-1)}=x y^{k(4 s-1)}=x y^{4 k s-k}=y^{k}\left(x y^{k}\right) y^{-k},
$$

so we need only consider (19). When $r=2 q+1$ we get

$$
y^{r} x y^{k} y^{-r}=x y^{k+r(n-2)}=x y^{k+(2 q+1)(n-2)}=x y^{k+2 q n+n-2-4 q}=x y^{k+4(s-q)-2}
$$

and for $r=2 p$ we get

$$
y^{r} x y^{k} y^{-r}=x y^{k+r(n-2)}=x y^{k+2 p(n-2)}=x y^{k-4 p} .
$$

Since $p$ and $q$ are arbitrary integers we get two conjugacy classes:

$$
\left\{x, x y^{2}, \ldots, x y^{2 n-2}\right\}, \quad\left\{x y, x y^{3}, \ldots, x y^{2 n-1}\right\} .
$$

Thus in the case of $n=4$ we get exactly $n+3$ conjugacy classes.

3.1.1. Representations of $\mathbb{U}_{n}, n$ even.

Proposition 3.1.2. When $n=2(2 l+1)$ the irreducible representations of $\mathbb{U}_{n}$ are the eight one dimensional irreducible representations $\rho_{1}$ (the trivial representation),

$$
\begin{gathered}
\rho_{2}(x)=1, \quad \rho_{2}(y)=-1, \quad \rho_{3}(x)=-1, \quad \rho_{3}(y)=-1, \quad \rho_{4}(x)=-1, \quad \rho_{4}(y)=1, \\
\rho_{5}(x)=1, \quad \rho_{5}(y)=\imath, \quad \rho_{6}(x)=1, \quad \rho_{6}(y)=-\imath, \quad \rho_{7}(x)=-1, \\
\rho_{7}(y)=-\imath, \quad \rho_{8}(x)=-1, \quad \rho_{8}(y)=\imath,
\end{gathered}
$$

and the two dimensional irreducible representations for $\zeta=\exp (2 \pi \imath / 2 n)$,

$$
R_{h}(x)=\left(\begin{array}{ll}
0 & 1 \\
1 & 0
\end{array}\right), \quad R_{h}(y)=\left(\begin{array}{cc}
\zeta^{h} & 0 \\
0 & (-1)^{h} \zeta^{-h}
\end{array}\right)
$$

for $h$ odd (excluding $h=n / 2$ if $n=2(2 l+1$ ) with $1 \leq h<n$ or $n<h<2 n$ and $h$ even with $2 \leq h<2 n, h \neq n$. Thus there are $8+(n / 2)-1+(n / 2)-1=n+6$ irreducible representations.

When $n=4 l$ the irreducible representations of $\mathbb{U}_{n}$ are the four one dimensional irreducible representations $\rho_{1}$ (the trivial representation):

$$
\rho_{2}(x)=1, \quad \rho_{2}(y)=-1, \quad \rho_{3}(x)=-1, \quad \rho_{3}(y)=-1, \quad \rho_{4}(x)=-1, \quad \rho_{4}(y)=1,
$$

and the two dimensional irreducible representations for $\zeta=\exp (2 \pi \imath / 2 n)$ given by (20). In this case, there are $4+(n / 2)+(n / 2)-1=n+3$ irreducible representations.

Note in the case $n=4 l$ above we have the sum of the squares of the dimensions of the irreducible representations with their multiplicities is $4+4(n-1)=4 n=\left|\mathbb{U}_{n}\right|$ as it should be and also the number of conjugacy classes $n+3$ is equal to the number of inequivalent irreducible representations. In the case $n=2(2 l+1)$ we have $n+6=8+(n-2)$ conjugacy classes and $8+(n-2)$ irreducible representations. Then we can check the sum of the squares of the dimensions of the irreducible representations with their multiplicities $8+4(n-2)=4 n$. 
Proof. For a 1-dimensional representation $\rho$ of $\mathbb{U}_{k}$ we must check the defining relations for $\mathbb{U}_{k}$ :

$$
\rho(x) \rho(y) \rho(x) \rho(y)^{n+1}=1=\rho(x)^{2}=\rho(y)^{2 n}
$$

where $\rho(x), \rho(y) \in \mathbb{C}^{\times}$. Thus $\rho(x)= \pm 1$ and from the first equation and last equations we get $\rho(y)^{n+2}=1=\rho(y)^{2 n}$ so $\rho(y)^{2 n+4}=\left(\rho(y)^{n+2}\right)^{2}=1$. Hence $\rho(y)^{4}=1$ and $\rho(y) \in\{ \pm 1, \pm \imath\}$. When $n=4 l$ we have $\rho(y)^{n}=\rho(y)^{4 l}=1=\rho(y)^{n+2}$ so that $\rho(y)^{2}=1$ and $\rho(y)= \pm 1$.

For $0 \leq h<2 n$ we have representations $R_{h}: \mathbb{U}_{n} \rightarrow \mathrm{GL}_{2}(\mathbb{C})$ as above since

$$
R_{h}(x)^{2}=\left(\begin{array}{ll}
0 & 1 \\
1 & 0
\end{array}\right)^{2}=I_{2}=R_{h}(y)^{2 n}=\left(\begin{array}{cc}
\zeta^{h} & 0 \\
0 & (-1)^{h} \zeta^{-h}
\end{array}\right)^{2 n},
$$

and

$$
\begin{aligned}
R_{h}(x) R_{h}(y) R_{h}(x) R_{h}(y)^{n+1} & =\left(\begin{array}{ll}
0 & 1 \\
1 & 0
\end{array}\right)\left(\begin{array}{cc}
\zeta^{h} & 0 \\
0 & (-1)^{h} \zeta^{-h}
\end{array}\right)\left(\begin{array}{ll}
0 & 1 \\
1 & 0
\end{array}\right)\left(\begin{array}{cc}
\zeta^{h} & 0 \\
0 & (-1)^{h} \zeta^{-h}
\end{array}\right)^{n+1} \\
& =\left(\begin{array}{cc}
0 & (-1)^{h} \zeta^{-h} \\
\zeta^{h} & 0
\end{array}\right)\left(\begin{array}{ll}
0 & 1 \\
1 & 0
\end{array}\right)\left(\begin{array}{cc}
\zeta^{h(n+1)} & 0 \\
0 & (-1)^{h(n+1)} \zeta^{-h(n+1)}
\end{array}\right) \\
& =\left(\begin{array}{cc}
0 & (-1)^{h} \zeta^{-h} \\
\zeta^{h} & 0
\end{array}\right)\left(\begin{array}{cc}
0 & 1 \\
1 & 0
\end{array}\right)\left(\begin{array}{cc}
(-1)^{h} \zeta^{h} & 0 \\
0 & \zeta^{-h}
\end{array}\right) \\
& =\left(\begin{array}{cc}
0 & (-1)^{h} \zeta^{-h} \\
\zeta^{h} & 0
\end{array}\right)\left(\begin{array}{cc}
0 & \zeta^{-h} \\
(-1)^{h} \zeta^{h} & 0
\end{array}\right) \\
& =I_{2}
\end{aligned}
$$

as $n$ is even. Consequently $R_{h}$ is a representation.

For $1 \leq h<n$ odd, the representations $R_{h}$ and $R_{n-h}$ are isomorphic with change of basis matrix

as

$$
T=\left(\begin{array}{cc}
0 & -1 \\
-1 & 0
\end{array}\right)
$$

$$
\left(\begin{array}{cc}
0 & -1 \\
-1 & 0
\end{array}\right)\left(\begin{array}{cc}
\zeta^{h} & 0 \\
0 & (-1)^{h} \zeta^{-h}
\end{array}\right)\left(\begin{array}{cc}
0 & -1 \\
-1 & 0
\end{array}\right)=\left(\begin{array}{cc}
(-1)^{h} \zeta^{-h} & 0 \\
0 & \zeta^{h}
\end{array}\right)=\left(\begin{array}{cc}
\zeta^{n-h} & 0 \\
0 & \zeta^{-n+h}
\end{array}\right)
$$

Similarly $R_{g}$ and $R_{3 n-g}$ are equivalent for odd $g$ with $n<g<2 n$. Note that when $h$ is even (21) gives us $R_{h}$ and $R_{2 n-h}$ are equivalent.

Let us show that $R_{h}$ and $R_{g}$ are not equivalent for $1 \leq h<n<g<2 n$ for odd $g$ and $h$. Indeed suppose that such an isomorphism exits. Then there exists a change of basis matrix

implementing this isomorphism.

$$
\left(\begin{array}{ll}
a & b \\
c & d
\end{array}\right)
$$

Simplifying for $h$ odd

$$
\left(\begin{array}{ll}
a & b \\
c & d
\end{array}\right)\left(\begin{array}{ll}
0 & 1 \\
1 & 0
\end{array}\right)=\left(\begin{array}{ll}
0 & 1 \\
1 & 0
\end{array}\right)\left(\begin{array}{ll}
a & b \\
c & d
\end{array}\right) \quad \text { and } \quad\left(\begin{array}{ll}
a & b \\
c & d
\end{array}\right)\left(\begin{array}{cc}
\zeta^{h} & 0 \\
0 & -\zeta^{-h}
\end{array}\right)=\left(\begin{array}{cc}
\zeta^{g} & 0 \\
0 & -\zeta^{-g}
\end{array}\right)\left(\begin{array}{ll}
a & b \\
c & d
\end{array}\right)
$$


gives us

$$
\left(\begin{array}{ll}
a & b \\
c & d
\end{array}\right)=\left(\begin{array}{ll}
a & b \\
b & a
\end{array}\right) \quad \text { and } \quad\left(\begin{array}{cc}
\zeta^{h} a & -\zeta^{-h} b \\
\zeta^{h} b & -\zeta^{-h} a
\end{array}\right)=\left(\begin{array}{cc}
\zeta^{g} a & \zeta^{g} b \\
-\zeta^{-g} b & -\zeta^{-g} a
\end{array}\right) .
$$

Thus $\zeta^{h-g} a=a$ and $\zeta^{h+g} b=-b$. If $a \neq 0$, then $h \equiv g \bmod 2 n$. If $a=0$, then $h+g \equiv n \bmod 2 n$ so that $h+g=(2 k+1) n$ but $h+g<n+2 n=3 n$. Thus $2 k+1=1$ and we get $h+g=n$ so that $g=n-h<n$, a contradiction.

Next we show that these representations are irreducible for $h$ odd when $n=4 s$. Otherwise say $R_{h}$ fixes a one dimensional space spanned by $(a, b)^{t}$. Then the only one dimensional subspaces fixed by $R_{h}(x)$ are spanned by

$$
\left(\begin{array}{r}
1 \\
\pm 1
\end{array}\right)
$$

On the other hand

$$
\left(\begin{array}{cc}
\zeta^{h} & 0 \\
0 & -\zeta^{-h}
\end{array}\right)\left(\begin{array}{l}
1 \\
1
\end{array}\right)=\left(\begin{array}{c}
\zeta^{h} \\
-\zeta^{-h}
\end{array}\right)=\zeta^{h}\left(\begin{array}{c}
1 \\
-\zeta^{-2 h}
\end{array}\right)
$$

which is an eigenvalue if and only if $\zeta^{2 h}=-1$ or $h=n / 2$. Now $h$ odd means that $n=2(2 s+1)$. If $n=4 s$, then there is no invariant vector. Similarly

$$
\left(\begin{array}{cc}
\zeta^{h} & 0 \\
0 & -\zeta^{-h}
\end{array}\right)\left(\begin{array}{c}
1 \\
-1
\end{array}\right)=\left(\begin{array}{c}
\zeta^{h} \\
\zeta^{-h}
\end{array}\right)=\zeta^{h}\left(\begin{array}{c}
1 \\
\zeta^{-2 h}
\end{array}\right)
$$

leads also to $n=2(2 s+1)$. As a consequent we have $(n / 2)-1$ irreducible representations $R_{h}, h$ odd, if $n=4 s$ and $n / 2$ if $n=4 s$.

Note that when $a$ is even (21) gives us $R_{a}$ and $R_{2 n-a}$ are equivalent. Moreover $R_{n}$ is reducible as

Simplifying for $1 \leq h, g<n$

$$
R_{n}(y)=\left(\begin{array}{cc}
\zeta^{n} & 0 \\
0 & (-1)^{n} \zeta^{-n}
\end{array}\right)=\left(\begin{array}{cc}
-1 & 0 \\
0 & -1
\end{array}\right)
$$

$\left(\begin{array}{ll}a & b \\ c & d\end{array}\right)\left(\begin{array}{ll}0 & 1 \\ 1 & 0\end{array}\right)=\left(\begin{array}{ll}0 & 1 \\ 1 & 0\end{array}\right)\left(\begin{array}{ll}a & b \\ c & d\end{array}\right) \quad$ and $\quad\left(\begin{array}{ll}a & b \\ c & d\end{array}\right)\left(\begin{array}{cc}\zeta^{2 h} & 0 \\ 0 & \zeta^{-2 h}\end{array}\right)=\left(\begin{array}{cc}\zeta^{2 g} & 0 \\ 0 & \zeta^{-2 g}\end{array}\right)\left(\begin{array}{ll}a & b \\ c & d\end{array}\right)$

give us

$$
\left(\begin{array}{ll}
a & b \\
c & d
\end{array}\right)=\left(\begin{array}{ll}
a & b \\
b & a
\end{array}\right) \quad \text { and } \quad\left(\begin{array}{ll}
\zeta^{2 h} a & \zeta^{-2 h} b \\
\zeta^{2 h} b & \zeta^{-2 h} a
\end{array}\right)=\left(\begin{array}{cc}
\zeta^{2 g} a & \zeta^{2 g} b \\
\zeta^{-2 g} b & \zeta^{-2 g} a
\end{array}\right) \text {. }
$$

Hence either $2 h=2 g \bmod 2 n$ or $2 h+2 g \equiv 0 \bmod 2 n$. In the first case $h-g=k n$ for some $k$. But $1 \leq h, g \leq n$ leads to $h=g$. If $h+g=n k$ for some $k$, then since $1 \leq g, h<n$ one gets $k=1$ and $g=n-h$ or $2 g=2 n-2 h$ which is the equivalence given above from (21).

Let us check that $R_{2 h}$ are irreducible for $2 \leq 2 h<n$. Otherwise $R_{2 h}$ fixes a one dimensional subspace spanned by either $(1,1)^{t}$ or $(1,-1)^{t}$. We calculate

$$
\left(\begin{array}{cc}
\zeta^{2 h} & 0 \\
0 & \zeta^{-2 h}
\end{array}\right)\left(\begin{array}{l}
1 \\
1
\end{array}\right)=\left(\begin{array}{c}
\zeta^{2 h} \\
\zeta^{-2 h}
\end{array}\right)=\zeta^{2 h}\left(\begin{array}{c}
1 \\
\zeta^{-4 h}
\end{array}\right)
$$

so that one would need $4 h=2 n$ or $2 h=n$. But $2 h<n$. 
Similarly

$$
\left(\begin{array}{cc}
\zeta^{2 h} & 0 \\
0 & \zeta^{-2 h}
\end{array}\right)\left(\begin{array}{c}
1 \\
-1
\end{array}\right)=\left(\begin{array}{c}
\zeta^{2 h} \\
-\zeta^{-2 h}
\end{array}\right)=\zeta^{2 h}\left(\begin{array}{c}
1 \\
-\zeta^{-4 h}
\end{array}\right)
$$

implies $4 h=2 n$ again a contradiction. Consequently there are $(n / 2)-1$ irreducible representations of the form $R_{2 h}$ with $2 \leq 2 h<n$.

Observe that $R_{h}, h$ odd, can not be equivalent to $R_{g}, g$ even as otherwise there is a change of basis matrix $A$ such that $A R_{h}(a) A^{-1}=R_{g}(a)$ for all $a \in \mathbb{U}_{n}$ which means in particular that $-1=\operatorname{det} R_{h}(y)=\operatorname{det} R_{g}(y)=1$.

3.2. Character Table and Irreducible Finite Dimensional Representations of $\mathbf{D i c}_{n}$. The dicyclic group is defined to be

$$
\mathbb{G}_{n}=\operatorname{Dic}_{n}:=\left\langle y, x: y^{2 n}=1, x^{2}=y^{n}, y x y=x\right\rangle .
$$

Proposition 3.2.1 ([Cox91]). The conjugacy classes of $\mathbb{G}_{n}$ are

(1) $\{1\},\left\{y^{n}\right\},\left\{y^{i}, y^{2 n-i}\right\} \quad$ where $1 \leq i \leq n-1$,

(2) $\left\{x, x y^{2}, \ldots, x^{2 n-2}\right\},\left\{x y, x y^{3}, \ldots, x y^{2 n-1}\right\}$.

Proof. From $y x y=x$, we have $x^{-1} y^{i} x=y^{-i}=x^{2 n-i}$. So we have the conjugacy classes in (11). Then we calculate

$$
x^{-1}\left(x y^{i}\right) x=y^{i} x=x y^{2 n-i} \quad \text { and } \quad y\left(x y^{i}\right) y^{-1}=y x y^{i-1}=x y^{i-2} .
$$

So we get two conjugacy classes in (2).

3.2.1. Irreducible representations. This section was certainly known to Coxeter in [Cox91. The dicyclic group $\mathrm{Dic}_{n}$ is as double cover of the dihedral group:

$$
\{ \pm 1\} \stackrel{y^{n}}{\longrightarrow} \mathrm{Dic}_{n} \longrightarrow D_{n} .
$$

3.3. When $n$ is even. We have $(n-2) / 2$ inequivalent irreducible representations $\sigma_{k}$ : $\mathrm{Dic}_{n} \rightarrow \mathrm{GL}_{2}(\mathbb{C})$ given by

$$
\sigma_{k}(a)=\left(\begin{array}{cc}
e^{\pi \imath k / n} & 0 \\
0 & e^{-\pi \imath k / n}
\end{array}\right), \quad \sigma_{k}(x)=\left(\begin{array}{ll}
0 & 1 \\
1 & 0
\end{array}\right)
$$

where $k=1,2, \ldots,(n-2) / 2$.

By Proposition 3.2 .1 one knows that there are $n+3$ conjugacy classes and thus there are $n+3$ irreducible representations (see [Ser77, Theorem 7, page 19]).

There are $n / 2$ irreducible representations of degree 2 of the form

$$
\tau_{k}(a)=\left(\begin{array}{cc}
e^{\pi \imath k / n} & 0 \\
0 & e^{-\pi \imath k / n}
\end{array}\right), \quad \tau_{k}(x)=\left(\begin{array}{cc}
0 & -1 \\
1 & 0
\end{array}\right)
$$

where $1 \leq k \leq n$ is odd.

In addition there is the trivial representation $\omega_{0}$ and the representations

$$
\begin{array}{ll}
\omega_{1}(y)=1, & \omega_{1}(x)=-1, \\
\omega_{2}(y)=-1, & \omega_{2}(x)=1, \\
\omega_{3}(y)=-1, & \omega_{3}(x)=-1 .
\end{array}
$$


3.4. When $n$ is odd. Again we describe $n+3$ irreducible representations over the complex numbers.

We have $(n-1) / 2$ inequivalent irreducible representations $\sigma_{k}: \operatorname{Dic}_{n} \rightarrow \mathrm{GL}_{2}(\mathbb{C})$ given by

$$
\sigma_{k}(a)=\left(\begin{array}{cc}
e^{\pi \imath k / n} & 0 \\
0 & e^{-\pi \imath k / n}
\end{array}\right), \quad \sigma_{k}(x)=\left(\begin{array}{ll}
0 & 1 \\
1 & 0
\end{array}\right)
$$

where $k=1,2, \ldots,(n-1) / 2$.

There are $(n-1) / 2$ other irreducible representations of degree 2 of the form

$$
\gamma_{k}(a)=\left(\begin{array}{cc}
e^{\pi \imath k / n} & 0 \\
0 & e^{-\pi \imath k / n}
\end{array}\right), \quad \gamma_{k}(x)=\left(\begin{array}{cc}
0 & -1 \\
1 & 0
\end{array}\right)
$$

where $k=1,2,3, \ldots, n-2$.

In addition there is the trivial representation $\omega_{0}$ and the representations

$$
\begin{array}{ll}
\omega_{1}(y)=-1, & \omega_{1}(x)=1, \\
\omega_{2}(y)=-1, & \omega_{1}(x)=\imath, \\
\omega_{3}(y)=-1, & \omega_{2}(x)=-\imath .
\end{array}
$$

\section{The DeCOMPosition OF THE SPACE OF KÄHLER DifFERENTiAls MOdUlO EXACT FORMS FOR $D_{2 k}$}

Let $r=2 n, R=R_{2}(p)$ and let $G:=\operatorname{Aut}(R)$ be the groups in Corollary 2.3. For $\phi \in G$ and $\overline{r d s} \in \Omega_{R} / d R$, the action of $G$ on the Kähler differential is given by:

$$
\phi(\overline{r d s})=\overline{\phi(r) d \phi(s)}
$$

First we include the following lemma for completeness.

Lemma 4.1 ([CI18], Lemma 7.1). If $n$ is even, the character table is given as follows:

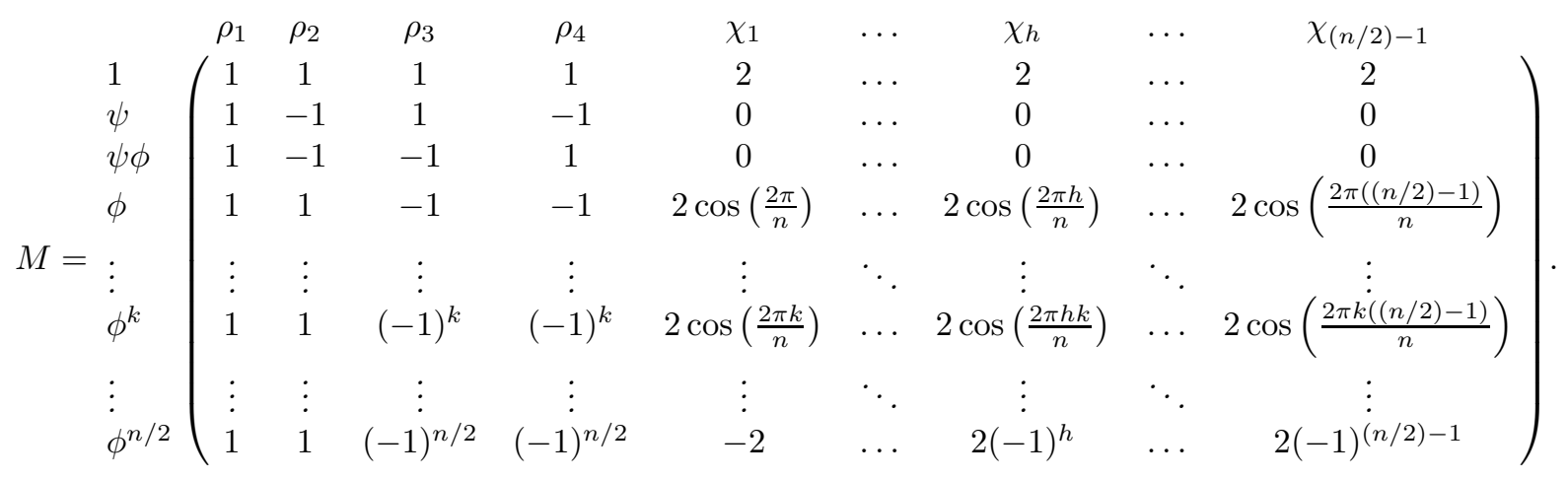


So we have

$$
\begin{aligned}
& M^{-1}=M^{t}\left(\begin{array}{ccccccccc}
\frac{1}{2 n} & 0 & 0 & 0 & \ldots & 0 & \ldots & \ldots & 0 \\
0 & \frac{n / 2}{2 n} & 0 & 0 & \ldots & 0 & \ldots & \ldots & 0 \\
0 & 0 & \frac{n / 2}{2 n} & 0 & \ldots & 0 & \ldots & \ldots & 0 \\
0 & 0 & 0 & \frac{2}{2 n} & \ldots & 0 & \ldots & \ldots & 0 \\
\vdots & \vdots & \vdots & \vdots & \ddots & \vdots & \ddots & \vdots & \vdots \\
0 & 0 & 0 & 0 & \ldots & \frac{2}{2 n} & \ldots & \vdots & 0 \\
\vdots & \vdots & \vdots & \vdots & \ddots & \vdots & \ddots & \vdots & \vdots \\
0 & 0 & 0 & 0 & \ldots & \vdots & \ldots & \frac{2}{2 n} & 0 \\
0 & 0 & 0 & 0 & \ldots & 0 & \ldots & 0 & \frac{1}{2 n}
\end{array}\right) \\
& =\left(\begin{array}{cccccccc}
\frac{1}{2 n} & \frac{1}{4} & \frac{1}{4} & \frac{1}{n} & \ldots & \frac{1}{n} & \ldots & \frac{1}{2 n} \\
\frac{1}{2 n} & -\frac{1}{4} & -\frac{1}{4} & \frac{1}{n} & \ldots & \frac{1}{n} \\
\frac{1}{2 n} & \frac{1}{4} & -\frac{1}{4} & -\frac{1}{n} & \ldots & \frac{(-1)^{k}}{n} & \ldots & \frac{(1}{2 n} \\
\frac{1}{2 n} & -\frac{1}{4} & \frac{1}{4} & -\frac{1}{n} & \ldots & \frac{(-1)^{k}}{n} & \ldots & \frac{(-1)^{n / 2}}{2 n} \\
\frac{1}{n} & 0 & 0 & \frac{2}{n} \cos (2 \pi / n) & \ldots & \frac{2}{n} \cos (2 \pi h / n) & \ldots & -\frac{1}{n} \\
\vdots & \vdots & \vdots & \vdots & \ddots & \vdots & \ddots & \vdots \\
\frac{1}{n} & 0 & 0 & \frac{2}{n} \cos (2 \pi h / n) & \ldots & \frac{2}{n} \cos (2 \pi h k / n) & \ldots & \frac{(-1)^{h}}{n} \\
\vdots & \vdots & \vdots & \vdots & \ddots & \vdots & \ddots & \vdots \\
\frac{1}{n} & 0 & 0 & \frac{2}{n} \cos (2 \pi((n / 2)-1) / n) & \ldots & \frac{2}{n} \cos (2 \pi k((n / 2)-1) / n) & \ldots & \frac{(-1)^{(n / 2)-1}}{n}
\end{array}\right) .
\end{aligned}
$$

Lemma 4.2. If $n$ is odd, the character table is given by the matrix

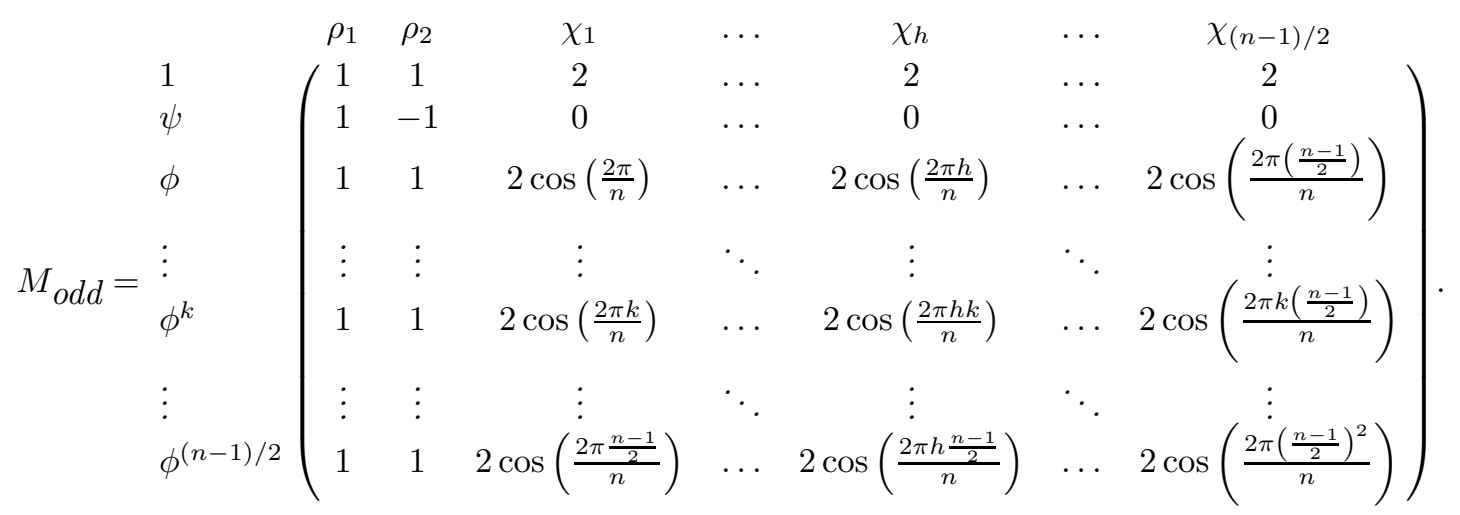

So

$$
M_{\text {odd }}^{-1}=M_{\text {odd }}^{t}\left(\begin{array}{ccccccccc}
\frac{1}{2 n} & 0 & 0 & 0 & \ldots & 0 & \ldots & \ldots & 0 \\
0 & \frac{n}{2 n} & 0 & 0 & \ldots & 0 & \ldots & \ldots & 0 \\
0 & 0 & \frac{2}{2 n} & 0 & \ldots & 0 & \ldots & \ldots & 0 \\
0 & 0 & 0 & \frac{2}{2 n} & \ldots & 0 & \ldots & \ldots & 0 \\
\vdots & \vdots & \vdots & \vdots & \ddots & \vdots & \ddots & \vdots & \vdots \\
0 & 0 & 0 & 0 & \ldots & \frac{2}{2 n} & \ldots & \ldots & 0 \\
\vdots & \vdots & \vdots & \vdots & \ddots & \vdots & \ddots & \vdots & \vdots \\
0 & 0 & 0 & 0 & \ldots & \ldots & \ldots & \frac{2}{2 n} & 0 \\
0 & 0 & 0 & 0 & \ldots & 0 & \ldots & 0 & \frac{2}{2 n}
\end{array}\right)
$$


THE CENTER OF HYPERELLIPTIC KRICHEVER-NOVIKOV ALGEBRAS

$$
=\left(\begin{array}{ccccccc}
\frac{1}{2 n} & \frac{n}{2 n} & \frac{2}{2 n} & \ldots & \frac{2}{2 n} & \ldots & \frac{2}{2 n} \\
\frac{1}{2 n} & -\frac{n}{2 n} & \frac{2}{2 n} & \ldots & \frac{2}{2 n} & \ldots & \frac{2}{2 n} \\
\frac{2}{2 n} & 0 & \frac{2}{2 n} \cos \left(\frac{2 \pi}{n}\right) & \ldots & \frac{2}{2 n} \cos \left(\frac{2 \pi k}{n}\right) & \ldots & \frac{2}{2 n} \cos \left(\frac{2 \pi \frac{n-1}{2}}{n}\right) \\
\vdots & \vdots & \vdots & \ddots & & \vdots & \vdots \\
\frac{2}{2 n} & 0 & \frac{2}{2 n} \cos \left(\frac{2 \pi h}{n}\right) & \ldots & \frac{2}{2 n} \cos \left(\frac{2 \pi h k}{n}\right) & \ldots & \frac{2}{2 n} \cos \left(\frac{2 \pi h \frac{n-1}{2}}{n}\right) \\
\vdots & \vdots & \vdots & \vdots & & \ddots & \vdots \\
\frac{2}{2 n} & 0 & \frac{2}{2 n} \cos \left(\frac{2 \pi\left(\frac{n-1}{2}\right)}{n}\right) & \ldots & \frac{2}{2 n} \cos \left(\frac{2 \pi k\left(\frac{n-1}{2}\right)}{n}\right) & \ldots & \frac{2}{2 n} \cos \left(\frac{2 \pi\left(\frac{n-1}{2}\right)^{2}}{n}\right)
\end{array}\right) .
$$

Proof. The matrix $M_{\text {odd }}$ is the character table for $D_{n}$ when $n$ is odd. From page 38 in [Ser77], we obtain the character table for $n$ odd:

\begin{tabular}{|c|c|c|}
\hline & $\left(\phi_{\zeta}^{+}\right)^{k}$ & $\psi_{c}^{+}\left(\phi_{\zeta}^{+}\right)^{k}$ \\
\hline$\rho_{1}$ & 1 & 1 \\
\hline$\rho_{2}$ & 1 & -1 \\
\hline$\chi_{h}$ & $2 \cos (2 \pi h k / n)$ & 0 \\
\hline
\end{tabular}

where $1 \leq h \leq(n-1) / 2, \psi_{c}^{+}$is a reflection, and $\phi_{\zeta}^{+}$is a rotation.

Letting $\Xi$ denote the set of conjugacy classes of the group $D_{n}$, we get the inverse matrix for $M_{\text {odd }}$ from the orthogonality of the characters of the irreducible representations. That is, one uses

$$
\sum_{\{g\} \in \Xi} \frac{|\{g\}|}{\left|D_{n}\right|} \chi_{\pi}(g) \overline{\chi_{\rho}(g)}= \begin{cases}1 & \text { for } \pi \cong \rho \\ 0 & \text { otherwise }\end{cases}
$$

for any two irreducible representations $\pi$ and $\rho$ of $D_{n}$ (see page 260 in [Ter99]).

The distinct conjugacy classes of $D_{n}$ (for $n$ odd) are:

$$
\{\mathrm{I}\},\left\{\left(\phi_{\zeta}^{+}\right),\left(\phi_{\zeta}^{+}\right)^{-1}\right\}, \ldots,\left\{\left(\phi_{\zeta}^{+}\right)^{i},\left(\phi_{\zeta}^{+}\right)^{-i}\right\}, \ldots,\left\{\left(\phi_{\zeta}^{+}\right)^{\widetilde{m}},\left(\phi_{\zeta}^{+}\right)^{-\widetilde{m}}\right\},\left\{\psi_{c}^{+}\left(\phi_{\zeta}^{+}\right)^{k}: 0 \leq k<n\right\}
$$

since odd dihedral groups have trivial center.

So under an action by $G$, we decompose $\Omega_{R} / d R=Z(\widehat{\mathfrak{g}})$ into a direct sum of irreducible representations. Our goal in this section is to describe the module structure of $\Omega_{R} / d R$ into irreducibles under the action by $G$ for a particular $R_{2}(p)$.

For completeness, we state the full result when the automorphism group is $D_{n}$, where $n \in \mathbb{N}$.

Theorem 4.3. Let $p(t)=t\left(t-\alpha_{1}\right) \cdots\left(t-\alpha_{2 n}\right)$, where $\alpha_{i}$ are pairwise distinct. Assume $\sigma_{c}^{ \pm}$exists in $\operatorname{Aut}\left(\mathcal{R}_{2}(p)\right)$ for some nonzero $c \in \mathbb{C}, c^{2 n}=a_{1}$ and $k \mid n$.

If $k$ is also even, then under the action of $D_{2 k}$ the center decomposes as:

$$
\Omega_{R} / d R \cong \mathbb{C} \omega_{0} \oplus \bigoplus_{i=3}^{4} U_{i}^{\frac{\left(1-(-1)^{k}\right) n}{2 k}} \oplus \bigoplus_{h=1}^{k-1} V_{h}^{\oplus \frac{\left(1-(-1)^{h}\right) n}{k}}
$$


where $U_{i}, i=1,2,3,4$ are the irreducible one dimensional representations for $D_{2 k}$ with character $\rho_{i}$ and $V_{h}$ are the irreducible 2-dimensional representations for $D_{2 k}$ with character $\chi_{h}, 1 \leq h \leq k-1$. Note $\mathbb{C} \omega_{0}$ and $U_{1}$ are the trivial representations.

If $k$ is odd, then the center decomposes as:

$$
\Omega_{R} / d R \cong \mathbb{C} \omega_{0} \oplus \bigoplus_{i=3}^{4} U_{i}^{\oplus \Upsilon_{i}\left(\epsilon_{i}, \nu_{i}\right)} \oplus \bigoplus_{j=1}^{k-1} V_{j}^{\oplus \frac{\left(1-(-1)^{j}\right) n}{k}}
$$

where

$$
\Upsilon_{i}\left(\epsilon_{i}, \nu_{i}\right)=\frac{n}{2 k}+\frac{\epsilon_{i}}{4} \sum_{i=n+3}^{2 n} c^{\frac{n+3-2 i}{2}} P_{i-n-3,-i}+\frac{\nu_{i}}{4} \sum_{i=n+3}^{2 n} \zeta^{\frac{2 n+3-2 i}{2}} c^{\frac{n+3-2 i}{2}} P_{i-n-3,-i}
$$

with

$\Upsilon_{i}\left(\epsilon_{i}, \nu_{i}\right)=\frac{\left(1-(-1)^{k}\right) n}{2 k}\left(\delta_{i, 3}+\delta_{i, 4}\right)+\frac{1-(-1)^{n}}{4}\left(\delta_{i, 4}-\delta_{i, 3}\right)+\frac{1}{2}(-1)^{i} \sum_{i=n+3}^{2 n} c^{n+3-2 i} P_{i-n-3,-i}$.

If the automorphism group is $D_{n}$, where $n$ is odd, then the center decomposes as

$$
\Omega_{R} / d R \cong \mathbb{C} \omega_{0} \oplus U_{1}^{\Xi_{1}} \oplus U_{2}^{\Xi_{2}} \oplus \bigoplus_{j=1}^{\frac{n-1}{2}} V_{j}
$$

where

$$
\Xi_{1}=\frac{1}{2}-\frac{1}{2} \sum_{i=n+3}^{2 n} c^{\frac{n+3-2 i}{2}} P_{i-n-3,-i} \quad \text { and } \quad \Xi_{2}=\frac{3}{2}+\frac{1}{2} \sum_{i=n+3}^{2 n} c^{\frac{n+3-2 i}{2}} P_{i-n-3,-i}
$$

$\mathbb{C} \omega_{0}$ is a 1-dimensional irreducible representation, $U_{i}$ are pairwise distinct 1-dimensional irreducible representations, and $V_{j}$ are pairwise distinct 2-dimensional irreducible representations.

When $n=2$, we have $\mathbb{C} \omega_{2} \oplus \mathbb{C} \omega_{3}$ and $\mathbb{C} \omega_{1} \oplus \mathbb{C} \omega_{4}$ as two 2-dimensional irreducible representations of $D_{2} \times \mathbb{Z}_{2}$.

Remark 4.4. If $\sigma_{c}^{ \pm}$does not exist, then we obtain the automorphism group being $C_{2 k}$, and the decomposition is done in Theorem 7.2.(1) in [CI18].

Corollary 4.5. If $\overline{\omega_{i}}=c^{-\frac{n+3-2 i}{2}} \zeta^{-\frac{i}{2}} \omega_{i}$ for $1 \leq i \leq n+2$, then $\overline{\omega_{i}}$ and $\overline{\omega_{n+3-i}}$ span a 2-dimensional irreducible representation.

If $n$ is even, then $1 \leq i \leq(n+2) / 2$ in Corollary 4.5,

We will now prove Theorem 4.3 .

Proof. When $n$ is even, the proof is given on pages 85-90 in [CI18]. 
For $n$ odd, we have $\mathbb{C} \omega_{i} \oplus \mathbb{C} \omega_{n+3-i}$ forming 2-dimensional irreducible $D_{n}$-representations for $1 \leq i \leq \frac{n+1}{2}$ since similar to the case when $n$ is even, we have

$$
\left.\phi_{\zeta}^{+}\right|_{\left\{\omega_{i}, \omega_{n+3-i}\right\}}=\left(\begin{array}{cc}
\zeta^{\frac{2 n+3-2 i}{2}} & 0 \\
0 & \zeta^{\frac{2 i-3}{2}}
\end{array}\right) \text { and }\left.\psi_{c}^{+}\right|_{\left\{\omega_{i}, \omega_{n+3-i}\right\}}=\left(\begin{array}{cc}
0 & -c^{-\frac{n+3-2 i}{2}} \\
-c^{n+3-2 i} & 0
\end{array}\right),
$$

where $\operatorname{tr}\left(\left.\phi_{\zeta}^{+}\right|_{\left\{\omega_{i}, \omega_{n+3-i}\right\}}\right)=\zeta^{n}$ and $\operatorname{tr}\left(\left.\psi_{c}^{+}\right|_{\left\{\omega_{i}, \omega_{n+3-i}\right\}}\right)=0$. It follows from Corollary 4.5 that we indeed have 2-dimensional irreducible representations.

Furthermore, since

$$
\psi_{c}^{+}\left(\omega_{\frac{n+3}{2}}\right)=-\omega_{\frac{n+3}{2}} \quad \text { and } \quad \phi_{\zeta}^{+}\left(\omega_{\frac{n+3}{2}}\right)=-\omega_{\frac{n+3}{2}},
$$

$\omega_{\frac{n+3}{2}}$ is a basis vector for a one-dimensional sign representation $\mathbb{C} \omega_{\frac{n+3}{2}}$ under the action of $\stackrel{2}{D_{n}}$ (for $n$ odd).

Similar to the proof of Theorem 7.2 in CI18, we have the automorphisms

$$
\phi_{\zeta}^{+}\left(\omega_{i}\right)=\zeta^{\frac{2 n+3-2 i}{2}} \omega_{i} \text { for } n+3 \leq i \leq 2 n
$$

and

$$
\psi_{c}^{+}\left(\omega_{i}\right)=-c^{n+3-2 i} \omega_{n+3-i}, \text { where } n+3 \leq i \leq 2 n .
$$

Since $\omega_{n+3-i}=\overline{t^{i-n-3} u d t}$,

$$
\overline{t^{i-n-3} u d t}=\sum_{k=1}^{2 n} P_{i-n-3,-k} \omega_{k} .
$$

Thus for $n+3 \leq i \leq 2 n$, we have

$$
\psi_{c}^{+}\left(\omega_{i}\right)=-c^{n+3-2 i} \sum_{k=1}^{2 n} P_{i-n-3,-k} \omega_{k} .
$$

The trace of $\phi_{\zeta}^{+}$in basis $\left\{\omega_{1}, \ldots, \omega_{2 n}\right\}$ is

$$
\operatorname{tr}\left(\phi_{\zeta}^{+}\right)=\sum_{i=1}^{2 n} \zeta^{\frac{2 n+3-2 i}{2}}
$$

while the trace of $\psi_{c}^{+}$with respect to $\left\{\omega_{1}, \ldots, \omega_{2 n}\right\}$ is

$$
\operatorname{tr}\left(\psi_{c}^{+}\right)=-1-\sum_{i=n+3}^{2 n} c^{n+3-2 i} P_{i-n-3,-i}
$$

For $n$ odd, the system of equations we need to solve is

$$
\chi_{\left(\Omega_{R} / d R\right) / \mathbb{C} \omega_{0}}=n_{1} \rho_{1}+n_{2} \rho_{2}+\sum_{h=1}^{\frac{n-1}{2}} m_{h} \chi_{h},
$$


which are precisely,

$$
\begin{gathered}
2 n=n_{1}+n_{2}+\sum_{h=1}^{\frac{n-1}{2}} 2 m_{h}, \\
\sum_{i=1}^{2 n} \zeta^{\frac{(2 n+3-2 i) k}{2}}=n_{1}+n_{2}+\sum_{h=1}^{\frac{n-1}{2}} 2 m_{h} \cos (2 \pi h k / n) \quad \text { for } 1 \leq k \leq \widetilde{m}=(n-1) / 2, \\
-1-\sum_{i=n+3}^{2 n} c^{n+3-2 i} P_{i-n-3,-i}=n_{1}-n_{2} \quad \text { for } \psi_{c}^{+} .
\end{gathered}
$$

The sum $\sum_{i=1}^{2 n} \zeta^{\frac{(2 n+3-2 i) k}{2}}$ simplifies as:

$$
\sum_{i=1}^{2 n} \zeta^{\frac{(2 n+3-2 i) k}{2}}=\zeta^{\frac{3}{2} k} \sum_{i=1}^{2 n} \zeta^{(n-i) k}=\zeta^{\frac{3}{2} k}\left(\zeta^{(n-1) k}+\zeta^{(n-2) k}+\ldots+\zeta^{k}+1+\zeta^{-k}+\ldots+\zeta^{-2 n k}\right)=0
$$

Using Equation (14), we see that $3 n-2 i+4=2 n-q k+1$, where $n+3 \leq i \leq \frac{3(n+1)}{2}$ and $0 \leq q \leq 2 n / k$. The equation simplifies as $n+3-2 i=-q k$. Since $n+3-2 i$ is even, $q k$ is also even.

So the above set of equations can be rewritten as:

$$
M_{\text {odd }}\left(\begin{array}{c}
n_{1} \\
n_{2} \\
m_{1} \\
m_{2} \\
\vdots \\
m_{h} \\
\vdots \\
m_{(n-1) / 2}
\end{array}\right)=\left(\begin{array}{c}
2 n \\
-1-\sum_{i=n+3}^{2 n} c^{n+3-2 i} P_{i-n-3,-i} \\
0 \\
0 \\
\vdots \\
0 \\
0
\end{array}\right)
$$

So

$$
\left(\begin{array}{c}
n_{1} \\
n_{2} \\
m_{1} \\
m_{2} \\
\vdots \\
m_{h} \\
\vdots \\
m_{(n-1) / 2}
\end{array}\right)=\left(\begin{array}{ccccccc}
\frac{1}{2 n} & \frac{n}{2 n} & \frac{2}{2 n} & \ldots & \frac{2}{2 n} & \ldots & \frac{2}{2 n} \\
\frac{2}{2 n} & -\frac{n}{2 n} & \frac{2}{2 n} \cos \left(\frac{2 \pi}{n}\right) & \ldots & \frac{2}{2 n} \cos \left(\frac{2 \pi k}{n}\right) & \ldots & \frac{2}{2 n} \cos \left(\frac{2 \pi \frac{n-1}{2}}{n}\right) \\
\vdots & \vdots & \vdots & \ddots & & \vdots & \vdots \\
\frac{2}{2 n} & 0 & \frac{2}{2 n} \cos \left(\frac{2 \pi h}{n}\right) & \ldots & \frac{2}{2 n} \cos \left(\frac{2 \pi h k}{n}\right) & \ldots & \frac{2}{2 n} \cos \left(\frac{2 \pi h \frac{n-1}{2}}{n}\right) \\
\vdots & \vdots & \vdots & \vdots & & \ddots & \vdots \\
\frac{2}{2 n} & 0 & \frac{2}{2 n} \cos \left(\frac{2 \pi\left(\frac{n-1}{2}\right)}{n}\right) & \ldots & \frac{2}{2 n} \cos \left(\frac{2 \pi k\left(\frac{n-1}{2}\right)}{n}\right) & \ldots & \frac{2}{2 n} \cos \left(\frac{2 \pi\left(\frac{n-1}{2}\right)^{2}}{n}\right)
\end{array}\right)\left(\begin{array}{c}
2 n \\
\Xi \\
0 \\
0 \\
\vdots \\
0 \\
0
\end{array}\right),
$$


where $\Xi=-1-\sum_{i=n+3}^{2 n} c^{n+3-2 i} P_{i-n-3,-i}$, which we conclude

$$
\left(\begin{array}{c}
n_{1} \\
n_{2} \\
m_{1} \\
m_{2} \\
\vdots \\
m_{h} \\
\vdots \\
m_{(n-1) / 2}
\end{array}\right)=\left(\begin{array}{c}
\frac{1}{2}-\frac{1}{2} \sum_{i=n+3}^{2 n} c^{n+3-2 i} P_{i-n-3,-i} \\
\frac{3}{2}+\frac{1}{2} \sum_{i=n+3}^{2 n} c^{n+3-2 i} P_{i-n-3,-i} \\
2 \\
2 \\
\vdots \\
2
\end{array}\right)
$$

Now, let $n=2$. Then under the action by $D_{2} \times \mathbb{Z}_{2}$, we have:

$$
\psi_{c}^{+}\left(\omega_{1}\right)=-c^{\frac{3}{2}} \omega_{4}, \quad \psi_{c}^{+}\left(\omega_{2}\right)=-c^{\frac{1}{2}} \omega_{3}, \quad \psi_{c}^{+}\left(\omega_{3}\right)=-c^{-\frac{1}{2}} \omega_{2}, \quad \psi_{c}^{+}\left(\omega_{4}\right)=-c^{-\frac{3}{2}} \omega_{1} .
$$

This implies with respect to the ordered basis $\left\{\omega_{2}, \omega_{3}\right\}$ and $\left\{\omega_{1}, \omega_{4}\right\}$, the matrix for $\psi_{c}^{+}$ is given by:

$$
\left.\psi_{c}^{+}\right|_{\left\{\omega_{2}, \omega_{3}\right\}}=\left(\begin{array}{cc}
0 & -c^{-\frac{1}{2}} \\
-c^{\frac{1}{2}} & 0
\end{array}\right) \quad \text { and }\left.\quad \psi_{c}^{+}\right|_{\left\{\omega_{1}, \omega_{4}\right\}}=\left(\begin{array}{cc}
0 & -c^{-\frac{3}{2}} \\
-c^{\frac{3}{2}} & 0
\end{array}\right)
$$

respectively.

Under the action by $\phi_{\zeta}^{+}$, we have:

$$
\phi_{\zeta}^{+}\left(\omega_{1}\right)=\zeta^{\frac{5}{2}} \omega_{1}, \quad \phi_{\zeta}^{+}\left(\omega_{2}\right)=\zeta^{\frac{3}{2}} \omega_{2}, \quad \phi_{\zeta}^{+}\left(\omega_{3}\right)=\zeta^{\frac{1}{2}} \omega_{3}, \quad \phi_{\zeta}^{+}\left(\omega_{4}\right)=\zeta^{-\frac{1}{2}} \omega_{4} .
$$

So

$$
\left.\phi_{\zeta}^{+}\right|_{\left\{\omega_{2}, \omega_{3}\right\}}=\left(\begin{array}{cc}
\zeta^{\frac{3}{2}} & 0 \\
0 & \zeta^{\frac{1}{2}}
\end{array}\right) \quad \text { and }\left.\quad \phi_{\zeta}^{+}\right|_{\left\{\omega_{1}, \omega_{4}\right\}}=\left(\begin{array}{cc}
\zeta^{\frac{5}{2}} & 0 \\
0 & \zeta^{-\frac{1}{2}}
\end{array}\right) .
$$

Using the representation theory of the dihedral group $D_{2}$ of order 4 , we see that $\mathbb{C} \omega_{2} \oplus \mathbb{C} \omega_{3}$ and $\mathbb{C} \omega_{1} \oplus \mathbb{C} \omega_{4}$ are two 2-dimensional irreducible representations of $D_{2} \times \mathbb{Z}_{2}$.

We will now prove Corollary 4.5 for completeness.

Proof. For $n$ even, we change the basis to $\overline{\omega_{i}}=c^{-\frac{n+3-2 i}{2}} \zeta^{-\frac{i}{2}} \omega_{i}$ for $1 \leq i \leq n+2$ to see that we have 2-dimensional irreducible representations. Since

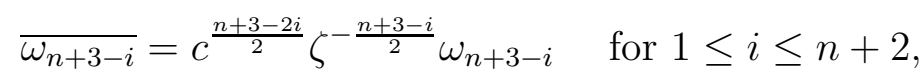

we have

(1) $\phi_{\zeta}^{+}\left(\overline{\omega_{i}}\right)=c^{-\frac{n+3-2 i}{2}} \zeta^{-\frac{2 i}{4}} \zeta^{\frac{2 n+3-2 i}{2}} \omega_{i}=\zeta^{\frac{2 n+3-2 i}{2}} \overline{\omega_{i}}$,

(2) $\phi_{\zeta}^{+}\left(\overline{\omega_{n+3-i}}\right)=c^{\frac{n+3-2 i}{2}} \zeta^{-\frac{n+3-i}{2}} \zeta^{\frac{-3+2 i}{4}} \omega_{n+3-i}=\zeta^{-\frac{2 n+3-2 i}{2}} \overline{\omega_{n+3-i}}$,

(3) $\psi_{c}^{+}\left(\bar{\omega}_{i}\right)=-c^{-\frac{n+3-2 i}{2}} \zeta^{-\frac{2 i}{4}} c^{n+3-2 i} \omega_{n+3-i}=\zeta^{\frac{2 n+3-2 i}{2}} \overline{\omega_{n+3-i}}$,

(4) $\psi_{c}^{+}\left(\overline{\omega_{n+3-i}}\right)=-c^{\frac{n+3-2 i}{2}} \zeta^{-\frac{n+3-i}{2}} c^{-\frac{n+3-2 i}{2}} \omega_{i}=\zeta^{-\frac{2 n+3-2 i}{2}} \overline{\omega_{i}}$. 
With respect to the basis $\overline{\omega_{1}}, \ldots, \overline{\omega_{n+2}}$, we obtain

$$
\left.\phi_{\zeta}^{+}\right|_{\left\{\overline{\omega_{i}}, \overline{\omega_{n+3-i}}\right\}}=\left(\begin{array}{cc}
\zeta^{\frac{2 n+3-2 i}{2}} & 0 \\
0 & \zeta^{-\frac{2 n+3-2 i}{2}}
\end{array}\right) \quad \text { and }\left.\quad \psi_{c}^{+}\right|_{\left\{\overline{\omega_{i}}, \overline{\omega_{n+3}-i}\right\}}=\left(\begin{array}{cc}
0 & \zeta^{-\frac{2 n+3-2 i}{2}} \\
\zeta^{\frac{2 n+3-2 i}{2}} & 0
\end{array}\right) \text {, }
$$

which coincide with classical 2-dimensional dihedral group irreducible representations.

For $n$ odd, with respect to the basis $\overline{\omega_{i}}=c^{-\frac{n+3-2 i}{4}} \zeta^{-\frac{i}{2}} \omega_{i}$ for $1 \leq i \leq n+2$, we have

$$
\left.\phi_{\zeta}^{+}\right|_{\left\{\overline{\omega_{i}}, \overline{\omega_{n+3-i}}\right\}}=\left(\begin{array}{cc}
\zeta^{\frac{2 n+3-2 i}{2}} & 0 \\
0 & \zeta^{-\frac{2 n+3-2 i}{2}}
\end{array}\right) \quad \text { and }\left.\quad \psi_{c}^{+}\right|_{\left\{\overline{\omega_{i}}, \overline{\omega_{n+3}-i}\right\}}=\left(\begin{array}{cc}
0 & \zeta^{-\frac{2 n+3-2 i}{2}} \\
\zeta^{\frac{2 n+3-2 i}{2}} & 0
\end{array}\right) \text {, }
$$

with

$$
\psi_{c}^{+}\left(\overline{\omega_{\frac{n+3}{2}}}\right)=-\overline{\omega_{\frac{n+3}{2}}} \quad \text { and } \quad \phi_{\zeta}^{+}\left(\overline{\omega_{\frac{n+3}{2}}}\right)=\zeta^{n / 2} \overline{\omega_{\frac{n+3}{2}}}=-\overline{\omega_{\frac{n+3}{2}}}
$$

The following examples are also in [CI18] but we state them here to provide examples for when $n$ is odd.

Example 4.6. Let $n=3$ and $k=3$. Then $p(t)=t\left(t^{3}-\alpha_{1}^{3}\right)\left(t^{3}-\alpha_{2}^{3}\right)$, with

$$
\psi_{c}^{+}=\left(\begin{array}{cccccc}
0 & 0 & 0 & 0 & -\frac{1}{c^{2}} & 0 \\
0 & 0 & 0 & -\frac{1}{c} & 0 & 0 \\
0 & 0 & -1 & 0 & 0 & 0 \\
0 & -c & 0 & 0 & 0 & 0 \\
-c^{2} & 0 & 0 & 0 & 0 & 0 \\
0 & 0 & 0 & 0 & 0 & -\frac{\alpha_{1}^{3} \alpha_{2}^{3}}{c^{6}}
\end{array}\right)
$$

In this case, $\alpha_{1}^{3} \alpha_{2}^{3}=c^{6}$, and the trace of $\psi_{c}^{+}$is -2 . This gives us multiplicities

$$
n_{1}=n_{2}=n_{3}=0, \quad n_{4}=2, \quad m_{1}=2 .
$$

Example 4.7. When $n=9$ and $k=3$, we obtain $\operatorname{tr}\left(\psi_{c}^{+}\right)=-2=-\operatorname{tr}\left(\psi_{c}^{+} \phi_{\xi}^{+}\right)$, and hence

$$
n_{1}=n_{2}=0, \quad n_{3}=2, \quad n_{4}=4, \quad m_{1}=6, \quad m_{2}=0 .
$$

Acknowledgments. B.C. is partially supported by a collaboration grant from the Simons Foundation \#319261. X.G. is partially supported by NSF of China (Grants 11101380, 11471294) and the Foundation for Young Teachers of Zhengzhou University (Grant 1421315071). K.Z. is partially supported by NSF of China (Grant 11271109) and NSERC.

\section{REFERENCES}

[Arb00] L. F. A. Arbogast, Du calcul des derivations.

[Bel28] E. T. Bell, Partition polynomials, Ann. of Math. (2) 29 (1927/28), no. 1-4, 38-46.

[BGG93] E. Bujalance, J. M. Gamboa, and G. Gromadzki, The full automorphism groups of hyperelliptic Riemann surfaces, Manuscripta Math. 79 (1993), no. 3-4, 267-282.

[Blo81] Spencer Bloch, The dilogarithm and extensions of Lie algebras, Algebraic $K$-theory, Evanston 1980 (Proc. Conf., Northwestern Univ., Evanston, Ill., 1980), Lecture Notes in Math., vol. 854, Springer, Berlin-New York, 1981, pp. 1-23. 
[Bre94] Murray Bremner, Universal central extensions of elliptic affine Lie algebras, J. Math. Phys. 35 (1994), no. 12, 6685-6692.

[Bre00] Thomas Breuer, Characters and automorphism groups of compact Riemann surfaces, London Mathematical Society Lecture Note Series, vol. 280, Cambridge University Press, Cambridge, 2000. MR 1796706 (2002i:14034)

[Cox91] H. S. M. Coxeter, Regular complex polytopes, second ed., Cambridge University Press, Cambridge, 1991.

[CF11] Ben Cox and Vyacheslav Futorny, DJKM algebras I: their universal central extension, Proc. Amer. Math. Soc. 35 (2011), no. 10, 3451-3460.

[CGLZ14] Ben Cox, Xiangqian Guo, Rencai Lu, and Kaiming Zhao, n-point Virasoro algebras and their modules of densities, Commun. Contemp. Math. 16 (2014), no. 3, 1350047, 27. MR 3211093

[CGLZ17] Ben Cox, Xiangqian Guo, Rencai Lu, and Kaiming Zhao, Simple superelliptic Lie algebras, Commun. Contemp. Math. 19 (2017), no. 3, 1650032, 22.

[Cox08] Ben Cox, Realizations of the four point affine Lie algebra $\mathfrak{s l}(2, R) \oplus\left(\Omega_{R} / d R\right)$, Pacific J. Math. 234 (2008), no. 2, 261-289.

[Cox16a] - Module structure of the center of the universal central extension of a genus zero Krichever-Novikov algebra, J. Algebra 467 (2016), 58-79. MR 3545955

[Cox16b] - On the universal central extension of hyperelliptic current algebras, Proc. Amer. Math. Soc. 144 (2016), no. 7, 2825-2835.

[CI18] Ben Cox and Mee Seong Im, On the module structure of the center of hyperelliptic KricheverNovikov algebras, Representations of Lie algebras, quantum groups and related topics, Contemp. Math., vol. 713, Amer. Math. Soc., Providence, RI, 2018, pp. 61-94.

[CZ17] Ben Cox and Kaiming Zhao, Certain families of polynomials arising in the study of hyperelliptic Lie algebras, The Ramanujan Journal, to appear, arXiv:1602.01432. (2017).

[FdB55] C. F. Faà di Bruno, Sullo sviluppo delle funzione, Ann. di Scienze Matem. et Fisiche di Tortoloni 6 (1855), 479-480.

[FdB57] _ Note sur un nouvelle formule de calcul différentiel, Quart. J. Math. 1 (1857), 359360.

[FH91] William Fulton and Joe Harris, Representation theory, Graduate Texts in Mathematics, vol. 129, Springer-Verlag, New York, 1991, A first course, Readings in Mathematics.

[Jor86] D. A. Jordan, On the ideals of a Lie algebra of derivations, J. London Math. Soc. (2) 33 (1986), no. 1, 33-39.

[Ser77] Jean-Pierre Serre, Linear representations of finite groups, Springer-Verlag, New YorkHeidelberg, 1977, Translated from the second French edition by Leonard L. Scott, Graduate Texts in Mathematics, Vol. 42.

[Sha03] Tanush Shaska, Determining the automorphism group of a hyperelliptic curve, Proceedings of the 2003 International Symposium on Symbolic and Algebraic Computation, ACM, New York, 2003, pp. 248-254.

[Skr88] S. M. Skryabin, Regular Lie rings of derivations, Vestnik Moskov. Univ. Ser. I Mat. Mekh. (1988), no. 3, 59-62.

[Skr04] Serge Skryabin, Degree one cohomology for the Lie algebras of derivations, Lobachevskii J. Math. 14 (2004), 69-107 (electronic).

[Tay04] Richard Taylor, Galois representations, Ann. Fac. Sci. Toulouse Math. (6) 13 (2004), no. 1, 73-119. MR 2060030 (2005a:11071)

[Ter99] Audrey Terras, Fourier analysis on finite groups and applications, London Mathematical Society Student Texts, vol. 43, Cambridge University Press, Cambridge, 1999. 
Department of Mathematics, College of Charleston, Charleston, SC 29424 USA

E-mail address: coxblecofc.edu

Department of Mathematics, Zhengzhou University, Zhengzhou 450001, Henan, P. R. CHINA

E-mail address: guoxq@zzu.edu.cn

Department of Mathematical Sciences, United States Military Academy, West Point, NY 10996 USA

E-mail address: meeseongimegmail.com

Department of Mathematics, Wilfrid Laurier University, Waterloo, On, Canada N2L 3C5, and College of Mathematics and Information Science, Hebei Normal (Teachers) University, Shijiazhuang, Hebei, 050016 P. R. China

E-mail address: kzhao@wlu.ca 